\title{
Isometric immersions into 3-dimensional homogeneous manifolds
}

\author{
Benoît Daniel
}

\begin{abstract}
We give a necessary and sufficient condition for a 2-dimensional Riemannian manifold to be locally isometrically immersed into a 3-dimensional homogeneous Riemannian manifold with a 4-dimensional isometry group. The condition is expressed in terms of the metric, the second fundamental form, and data arising from an ambient Killing field. This class of 3-manifolds includes in particular the Berger spheres, the Heisenberg group $\mathrm{Nil}_{3}$, the universal cover of the Lie group PSL $2(\mathbb{R})$ and the product spaces $\mathbb{S}^{2} \times \mathbb{R}$ and $\mathbb{H}^{2} \times \mathbb{R}$. We give some applications to constant mean curvature (CMC) surfaces in these manifolds; in particular we prove the existence of a generalized Lawson correspondence, i.e., a local isometric correspondence between CMC surfaces in homogeneous 3-manifolds.
\end{abstract}

Mathematics Subject Classification (2000). Primary: 53C42. Secondary: 53A35, 53B25.

Keywords. Isometric immersions, constant mean curvature surfaces, homogeneous manifolds, Gauss and Codazzi equations.

\section{Introduction}

A classical problem in geometry is to determine whether a Riemannian manifold $\mathcal{V}$ can be isometrically immersed in another Riemaniann manifold $\overline{\mathcal{V}}$. We will restrict ourselves to the case of codimension 1 immersions, i.e., $\mathcal{V}$ has dimension $n$ and $\overline{\mathcal{V}}$ has dimension $n+1$.

It is well known that the Gauss and Codazzi equations are necessary conditions relating the Riemann curvature tensor $\overline{\mathrm{R}}$ of $\overline{\mathcal{V}}$, the Riemann curvature tensor R of $\mathcal{V}$ and the shape operator $\mathrm{S}$ of $\mathcal{V}$. Denoting by $\nabla$ the Riemannian connection of $\mathcal{V}$, these equations are the following:

$$
\begin{gathered}
\langle\mathrm{R}(X, Y) Z, W\rangle-\langle\overline{\mathrm{R}}(X, Y) Z, W\rangle=\langle\mathrm{S} X, Z\rangle\langle\mathrm{S} Y, W\rangle-\langle\mathrm{S} Y, Z\rangle\langle\mathrm{S} X, W\rangle \\
\nabla_{X} \mathrm{~S} Y-\nabla_{Y} \mathrm{~S} X-\mathrm{S}[X, Y]=\overline{\mathrm{R}}(X, Y) N,
\end{gathered}
$$

for all vector fields $X, Y, Z$ and $W$ on $\mathcal{V}$.

Moreover, in the case where $\overline{\mathcal{V}}$ is a space-form, i.e., the sphere $\mathbb{S}^{n+1}$, the Euclidean space $\mathbb{R}^{n+1}$ or the hyperbolic space $\mathbb{H}^{n+1}$, the Gauss and Codazzi equations are also 
a sufficient condition for $\mathcal{V}$ to be locally isometrically immersed in $\overline{\mathcal{V}}$ with $\mathrm{S}$ as shape operator. In this case the Gauss and Codazzi equations involve only the metric and the shape operator of $\mathcal{V}$.

The author studied this problem when $\overline{\mathcal{V}}$ is a product manifold $\mathbb{S}^{n} \times \mathbb{R}$ or $\mathbb{H}^{n} \times \mathbb{R}$ ([Dan04]). Then the Gauss and Codazzi equations involve the metric of $\mathcal{V}$, its shape operator $\mathrm{S}$, the projection $T$ of the vertical vector field (i.e., the unit vector field corresponding to the factor $\mathbb{R}$ ) on the tangent space of $\mathcal{V}$ and the normal component $v$ of the vertical vector field (i.e., its inner product with the unit normal of $\mathcal{V}$ ). The author proved that the Gauss and Codazzi equations, together with two other compatibility equations coming from the fact that the vertical vector field is parallel, are a necessary and sufficient condition for $\mathcal{V}$ to be locally isometrically immersed in $\overline{\mathcal{V}}$ with $\mathrm{S}$ as shape operator, $T$ as tangent projection of the vertical vector field and $v$ as normal component of the vertical vector field.

It is natural to try to generalize this result to other homogeneous Riemannian manifolds. We will investigate the case of surfaces in manifolds of dimension 3, i.e., $n=2$. Indeed, the classification of simply connected 3-dimensional homogeneous manifolds is well known. Such a manifold has an isometry group of dimension 3, 4 or 6 . When the dimension of the isometry group is 6 , then we have a space form. When the dimension of the isometry group is 3 , the manifold has the geometry of the Lie group $\mathrm{Sol}_{3}$.

In this paper we will consider the homogeneous manifolds whose isometry groups have dimension 4: such a manifold is a Riemannian fibration over a 2-dimensional space form, the fibers are geodesics and there exists a one-parameter family of translations along the fibers, generated by a unit Killing field $\xi$ which will be called the vertical vector field. These manifolds are classified, up to isometry, by the curvature $\kappa$ of the base surface of the fibration and the bundle curvature $\tau$, where $\kappa$ and $\tau$ can be any real numbers satisfying $\kappa \neq 4 \tau^{2}$. The bundle curvature is the number $\tau$ such $\bar{\nabla}_{X} \xi=\tau X \times \xi$ for any vector field $X$ on $\overline{\mathcal{V}}$, where $\bar{\nabla}$ denotes the Riemannian connection of $\overline{\mathcal{V}}$.

When the bundle curvature $\tau$ vanishes (and then $\kappa \neq 0$ ), we get a product manifold $\mathbb{M}^{2}(\kappa) \times \mathbb{R}$ where $\mathbb{M}^{2}(\kappa)$ is the simply connected 2-manifold of constant curvature $\kappa$. Their isometry group has 4 connected components. The vertical vector $\xi$ is simply the vector corresponding to the factor $\mathbb{R}$. This case was treated in [Dan04].

When $\tau \neq 0$, the isometry group has 2 connected components: an isometry either preserves the orientations of both the fibers and the base of the fibration, or reverses both orientations. These manifolds are of three types: they have the isometry group of the Berger spheres for $\kappa>0$, of the Heisenberg group $\mathrm{Nil}_{3}$ for $\kappa=0$, and of $\widetilde{\mathrm{PSL}_{2}(\mathbb{R})}$ for $\kappa<0$. In this paper we will deal with these three types of manifold. Like for $\mathbb{M}^{2}(\kappa) \times \mathbb{R}$, the Gauss and Codazzi equations involve the metric of $\mathcal{V}$, its shape operator $\mathrm{S}$, the tangential projection $T$ of $\xi$ and the normal component $v$ of $\xi$. 
Denoting by $K$ the curvature of $\mathrm{d} s^{2}$, these equations become

$$
\begin{gathered}
K=\operatorname{det} \mathrm{S}+\tau^{2}+\left(\kappa-4 \tau^{2}\right) \nu^{2}, \\
\nabla_{X} \mathrm{~S} Y-\nabla_{Y} \mathrm{~S} X-\mathrm{S}[X, Y]=\left(\kappa-4 \tau^{2}\right) \nu(\langle Y, T\rangle X-\langle X, T\rangle Y)
\end{gathered}
$$

The first theorem is the following one.

Theorem (Theorem 4.3). Let $\mathcal{V}$ be a simply connected oriented Riemannian manifold of dimension $2, \mathrm{~d} s^{2}$ its metric (which we also denote by $\langle\cdot, \cdot\rangle$ ), $\nabla$ its Riemannian connection and $\mathrm{J}$ the rotation of angle $\frac{\pi}{2}$ on $\mathrm{T} \mathcal{V}$. Let $\mathrm{S}$ be a field of symmetric operators $\mathrm{S}_{y}: \mathrm{T}_{y} \mathcal{V} \rightarrow \mathrm{T}_{y} \mathcal{V}, T$ a vector field on $\mathcal{V}$ and $\mathcal{v}$ a smooth function on $\mathcal{V}$ such that $\|T\|^{2}+v^{2}=1$.

Let $\mathbb{E}$ be a 3-dimensional homogeneous manifold with a 4-dimensional isometry group and $\xi$ its vertical vector field. Let $\kappa$ be its base curvature and $\tau$ its bundle curvature. Then there exists an isometric immersion $f: \mathcal{V} \rightarrow \mathbb{E}$ such that the shape operator with respect to the normal $N$ associated to $f$ is

$$
\mathrm{d} f \circ \mathrm{S} \circ \mathrm{d} f^{-1}
$$

and such that

$$
\xi=\mathrm{d} f(T)+v N
$$

if and only if $\left(\mathrm{d} s^{2}, \mathrm{~S}, T, v\right)$ satisfies the Gauss and Codazzi equations for $\mathbb{E}$ and, for all vector fields $X$ on $\mathcal{V}$, the following equations:

$$
\nabla_{X} T=v(\mathbf{S} X-\tau \mathrm{J} X), \quad \mathrm{d} v(X)+\langle\mathrm{S} X-\tau \mathrm{J} X, T\rangle=0 .
$$

In this case, the immersion is unique up to a global isometry of $\mathbb{E}$ preserving the orientations of both the fibers and the base of the fibration.

The two additional conditions come from the fact that $\bar{\nabla}_{X} \xi=\tau X \times \xi$ for all vector fields $X$. We notice that this theorem seems specific to dimension 2 , since the operator of rotation $\mathrm{J}$ is involved.

The method to prove this theorem is similar to that of [Dan04] and was inspired by that of Tenenblat ([Ten71]): it is based on differential forms, moving frames and integrable distributions. However, things are technically much more complicated here: in [Dan04] the proof was simplified by the fact that $\mathbb{S}^{n} \times \mathbb{R}$ and $\mathbb{H}^{n} \times \mathbb{R}$ can be included in $\mathbb{R}^{n+2}$ and in the Lorentz space $\mathbb{L}^{n+2}$ respectively. We will first present the models used for the 3 -dimensional homogeneous manifolds, and then we will prove the theorem.

Finally we will give two applications of this theorem to constant mean curvature (CMC) surfaces in 3-dimensional homogeneous manifolds with 4-dimensional 
isometry group. There were many recent developments in this topic (see for example [FMP99], [NR04] and references therein); in particular Abresch and Rosenberg proved the existence of a holomorphic quadratic differential for CMC surfaces in these manifolds ([AR04], [AR05]).

The first application (Section 5.1) is the existence of an isometric correspondence between certain CMC surfaces in homogeneous 3-manifolds with the same "anisotropy coefficient" $\kappa-4 \tau^{2}$. This correspondence generalizes the classical Lawson correspondence between certain CMC surfaces in space-forms. This is the following theorem.

Theorem (see Theorem 5.2). Let $\mathbb{E}_{1}$ and $\mathbb{E}_{2}$ be two 3-dimensional homogeneous manifolds with 4-dimensional isometry groups, of base curvatures $\kappa_{1}$ and $\kappa_{2}$ and bundle curvatures $\tau_{1}$ and $\tau_{2}$ respectively, and such that

$$
\kappa_{1}-4 \tau_{1}^{2}=\kappa_{2}-4 \tau_{2}^{2} .
$$

Let $H_{1}$ and $H_{2}$ be two real numbers such that

$$
\tau_{1}^{2}+H_{1}^{2}=\tau_{2}^{2}+H_{2}^{2} .
$$

Then there exists an isometric correspondence between simply connected CMC $H_{1}$ surfaces in $\mathbb{E}_{1}$ and simply connected $C M C H_{2}$ surfaces in $\mathbb{E}_{2}$.

This correspondence is called the correspondence of the sister surfaces.

Moreover, one surface is obtained from the other one by rotating the traceless part of its shape operator by a fixed angle $\theta$ (depending on the $\tau_{j}$ and the $H_{j}$ ).

In particular we get a local isometric correspondence between minimal surfaces in the Heisenberg group $\mathrm{Nil}_{3}$ (with its standard metric) and CMC $\frac{1}{2}$ surfaces in $\mathbb{H}^{2} \times \mathbb{R}$. In this case we have $\theta=\frac{\pi}{2}$, which makes this correspondence similar to the conjugate cousin correspondence in space forms (see [GBKS03], [Kar05]). We compute some examples: the sister surface of the rotational minimal surface of equation $z=0$ in $\mathrm{Nil}_{3}$ is a graph over $\mathbb{H}^{2}$ in $\mathbb{H}^{2} \times \mathbb{R}$ invariant by a vertical rotation; the sister surface of the translational minimal surface of equation $z=\frac{x y}{2}$ in $\mathrm{Nil}_{3}$ is a graph over $\mathbb{H}^{2}$ in $\mathbb{H}^{2} \times \mathbb{R}$ invariant by a hyperbolic translation.

The second application (Section 5.3) is the existence of "twin immersions" of nonminimal CMC immersions in homogeneous 3-manifolds with non-vanishing bundle curvature. This twin immersion might be useful to prove an Alexandrov-type theorem in these manifolds.

Conventions and notations. In this paper we will use the following index conventions: Latin letters $i, j$, etc, denote integers between 1 and $n$ (or the integers 1 and 2), Greek letters $\alpha, \beta$, etc., denote integers between 1 and $n+1$ (or between 1 and 3). 
The set of vector fields on a Riemannian manifold $\mathcal{V}$ will be denoted by $\mathfrak{X}(\mathcal{V})$.

The Riemann curvature tensor $\mathrm{R}$ of a Riemannian manifold $\mathcal{V}$ of Riemannian connection $\nabla$ is defined using the following convention:

$$
\mathrm{R}(X, Y) Z=\nabla_{Y} \nabla_{X} Z-\nabla_{X} \nabla_{Y} Z+\nabla_{[X, Y]} Z .
$$

The shape operator of a hypersurface $\mathcal{V}$ of a Riemannian manifold $\overline{\mathcal{V}}$ associated to its unit normal $N$ is

$$
\mathrm{S} X=-\bar{\nabla}_{X} N
$$

where $\bar{\nabla}$ is the Riemannian connection of $\overline{\mathcal{V}}$.

\section{3-dimensional homogeneous manifolds with 4-dimensional isometry group}

In this section we will give the general setting for simply connected homogeneous 3-manifolds with 4-dimensional isometry group and we will describe the models used. We will consider only those having non-vanishing bundle curvature (since the product manifolds $\mathbb{M}^{2}(\kappa) \times \mathbb{R}$ were treated in [Dan04]). The reader can refer to [Sco83] for the geometry of 3-dimensional homogeneous manifolds.

2.1. Canonical frame. Let $\mathbb{E}$ be a simply connected 3 -dimensional homogeneous manifold with a 4-dimensional isometry group. Such a manifold is a Riemannian fibration over a simply connected 2-manifold of constant curvature $\kappa$. The fibers are geodesics. We will denote by $\xi$ a unit vector field on $\mathbb{E}$ tangent to the fibers; it will be called the vertical vector field. It is a Killing field (corresponding to translations along the fibers).

We will denote by $\bar{\nabla}$ and $\overline{\mathrm{R}}$ the Riemannian connection and the Riemannian curvature tensor of $\mathbb{E}$ respectively.

We assume that $\mathbb{E}$ is not a product manifold $\mathbb{M}^{2}(\kappa) \times \mathbb{R}$.

The manifold $\mathbb{E}$ locally has a direct orthonormal frame $\left(E_{1}, E_{2}, E_{3}\right)$ with

$$
E_{3}=\xi
$$

whose non-vanishing Christoffel symbols $\bar{\Gamma}_{\beta \gamma}^{\alpha}=\left\langle\bar{\nabla}_{E_{\beta}} E_{\gamma}, E_{\alpha}\right\rangle$ are the following:

$$
\begin{gathered}
\bar{\Gamma}_{12}^{3}=\bar{\Gamma}_{23}^{1}=-\bar{\Gamma}_{21}^{3}=-\bar{\Gamma}_{13}^{2}=\tau, \\
\bar{\Gamma}_{32}^{1}=-\bar{\Gamma}_{31}^{2}=\tau-\sigma,
\end{gathered}
$$

for some real numbers $\sigma$ and $\tau \neq 0$ (this will be made explicit in the sequel). Then we have

$$
\left[E_{1}, E_{2}\right]=2 \tau E_{3}, \quad\left[E_{2}, E_{3}\right]=\sigma E_{1}, \quad\left[E_{3}, E_{1}\right]=\sigma E_{2} .
$$


We will call $\left(E_{1}, E_{2}, E_{3}\right)$ the canonical frame of $\mathbb{E}$. For all vector field $X$ we have

$$
\bar{\nabla}_{X} E_{3}=\tau X \times E_{3}
$$

where $\times$ denotes the vector product in $\mathbb{E}$, i.e., for all vector fields $X, Y, Z,\langle X \times Y, Z\rangle=$ $\operatorname{det}_{\left(E_{1}, E_{2}, E_{3}\right)}(X, Y, Z)$.

Setting

$$
\langle\overline{\mathrm{R}}(X \wedge Y), Z \wedge W\rangle=\langle\overline{\mathrm{R}}(X, Y) Z, W\rangle,
$$

the matrix of $\overline{\mathrm{R}}$ in the basis $\left(E_{2} \wedge E_{3}, E_{3} \wedge E_{1}, E_{1} \wedge E_{2}\right)$ is

$$
\overline{\mathrm{R}}=\operatorname{diag}(a, a, b)
$$

with

$$
a=\tau^{2}, \quad b=-3 \tau^{2}+2 \sigma \tau .
$$

We now compute the curvature $\kappa$ of the base of the fibration. If $\bar{M} \rightarrow M$ is a Riemannian submersion, then the sectional curvature of a 2-plane $\Pi$ in $M$ generated by an orthonormal pair $(X, Y)$ is

$$
K(\Pi)=\bar{K}(\bar{\Pi})+\frac{3}{4}\left\|[\bar{X}, \bar{Y}]^{\mathrm{v}}\right\|^{2}
$$

where $\bar{X}$ and $\bar{Y}$ are horizontal lifts of $X$ and $Y$ in $\bar{M}, \bar{K}(\bar{\Pi})$ is the sectional curvature of a 2-plane $\bar{\Pi}$ in $\bar{M}$ generated by $(\bar{X}, \bar{Y})$, and where $Z^{\mathrm{v}}$ denotes the vertical part of a vector field $Z$ in $\bar{M}$ (see [Car92], chapter 8 ). In our case we get

$$
\kappa=\left\langle\overline{\mathrm{R}}\left(E_{1}, E_{2}\right) E_{1}, E_{2}\right\rangle+\frac{3}{4}\left\|\left[E_{1}, E_{2}\right]^{\mathrm{v}}\right\|^{2}=b+\frac{3}{4}\left\|2 \tau E_{3}^{\mathrm{v}}\right\|^{2}=b+3 \tau^{2} .
$$

Thus we have $b=\kappa-3 \tau^{2}$, and so

$$
\sigma=\frac{\kappa}{2 \tau}
$$

Proposition 2.1. For all vector fields $X, Y, Z, W$ on $\mathbb{E}$ we have

$$
\langle\overline{\mathrm{R}}(X, Y) Z, W\rangle=\left(\kappa-3 \tau^{2}\right)\left\langle\mathrm{R}_{0}(X, Y) Z, W\right\rangle+\left(\kappa-4 \tau^{2}\right)\left\langle\mathrm{R}_{1}(\xi ; X, Y) Z, W\right\rangle
$$

with

$$
\begin{gathered}
\mathrm{R}_{0}(X, Y) Z=\langle X, Z\rangle Y-\langle Y, Z\rangle X, \\
\mathrm{R}_{1}(V ; X, Y) Z=\langle Y, V\rangle\langle Z, V\rangle X+\langle Y, Z\rangle\langle X, V\rangle V \\
-\langle X, Z\rangle\langle Y, V\rangle V-\langle X, V\rangle\langle Z, V\rangle Y .
\end{gathered}
$$


Proof. We set $X=\tilde{X}+x \xi$ with $\tilde{X}$ horizontal and $x=\langle X$, $\xi\rangle$, etc. Using the multilinearity of the Riemann curvature tensor, we get a sum of 16 terms; the terms where $\xi$ appears three or four times, or twice at positions 1,2 or 3, 4, vanish by antisymmetry. The terms where $\xi$ appears once vanish because the matrix of $\overline{\mathrm{R}}$ in the basis $\left(E_{2} \wedge E_{3}, E_{3} \wedge E_{1}, E_{1} \wedge E_{2}\right)$ is diagonal. Hence we have

$$
\begin{aligned}
&\langle\overline{\mathrm{R}}(X, Y) Z, W\rangle=\langle\overline{\mathrm{R}}(\tilde{X}, \tilde{Y}) \tilde{Z}, \tilde{W}\rangle+y w\langle\overline{\mathrm{R}}(\tilde{X}, \xi) \tilde{Z}, \xi\rangle+y z\langle\overline{\mathrm{R}}(\tilde{X}, \xi) \xi, \tilde{W}\rangle \\
&+x w\langle\overline{\mathrm{R}}(\xi, \tilde{Y}) \tilde{Z}, \xi\rangle+x z\langle\overline{\mathrm{R}}(\xi, \tilde{Y}) \xi, \tilde{W}\rangle \\
&=\left(\kappa-3 \tau^{2}\right)(\langle\tilde{X}, \tilde{Z}\rangle\langle\tilde{Y}, \tilde{W}\rangle-\langle\tilde{X}, \tilde{W}\rangle\langle\tilde{Y}, \tilde{Z}\rangle) \\
&+\tau^{2}(y w\langle\tilde{X}, \tilde{Z}\rangle-y z\langle\tilde{X}, \tilde{W}\rangle-x w\langle\tilde{Y}, \tilde{Z}\rangle+x z\langle\tilde{Y}, \tilde{W}\rangle) \\
&=\left.\kappa-3 \tau^{2}\right)(\langle X, Z\rangle\langle Y, W\rangle-\langle X, W\rangle\langle Y, Z\rangle) \\
&-\left(\kappa-4 \tau^{2}\right)(\langle X, Z\rangle\langle Y, \xi\rangle\langle W, \xi\rangle+\langle Y, W\rangle\langle X, \xi\rangle\langle Z, \xi\rangle \\
&-\langle X, W\rangle\langle Y, \xi\rangle\langle Z, \xi\rangle-\langle Y, Z\rangle\langle X, \xi\rangle\langle W, \xi\rangle) .
\end{aligned}
$$

2.2. The manifolds with the isometry group of the Berger spheres. They occur when $\tau \neq 0$ and $\kappa>0$; they are fibrations over round 2-spheres. They are obtained by deforming the metric of a round sphere in a way preserving the Hopf fibration but modifying the length of the fibers. Their isometry group is included in that of the round sphere. The reader can refer to [Pet98].

The sphere $\mathbb{S}^{3}$ is the universal covering of $\mathrm{SO}_{3}(\mathbb{R})$, which can be identified with the unitary tangent bundle to the 2-sphere $\mathrm{US}^{2}$. Indeed, the group $\mathrm{SO}_{3}(\mathbb{R})$ acts transitively on $\mathrm{US}^{2}$, and the stabilizer of any point in $\mathrm{US}^{2}$ is trivial. The unitary tangent bundle $\mathrm{US}^{2}$ can be endowed with the metric induced by the standard metric on the tangent bundle $\mathbb{T S}^{2}$. We will give an expression of this metric.

Let $(x, y) \mapsto \varphi(x, y)$ be a conformal parametrization of a domain $D$ in $\mathbb{S}^{2}$ and let $\lambda$ be the conformal factor, i.e., the metric of $D$ is given by $\lambda^{2}\left(\mathrm{~d} x^{2}+\mathrm{d} y^{2}\right)$. Then a parametrization of $\mathrm{U} D$ is the following:

$$
(x, y, \theta) \mapsto\left(\varphi(x, y), \frac{1}{\lambda}\left(\cos \theta \partial_{x}+\sin \theta \partial_{y}\right)\right) .
$$

Let $p=\varphi(x, y) \in D, v \in \mathrm{T}_{p} D$ and $V \in \mathrm{T}_{(p, v)}(\mathrm{U} D)$. Let $\alpha(t)=(p(t), v(t))$ be a curve such that $v(t) \in \mathrm{T}_{p(t)} \mathbb{H}^{2}, p(0)=p, v(0)=v$ and $\alpha^{\prime}(0)=V$. Then the norm of $V$ is given by

$$
\|V\|_{(p, v)}^{2}=\|\mathrm{d} \pi(V)\|_{p}^{2}+\left\|\frac{\mathrm{D} v}{\mathrm{~d} t}(0)\right\|_{p}^{2}
$$

where $\pi: U D \rightarrow D$ is the canonical projection. 
We set $\alpha(t)=(x(t), y(t), \theta(t))$. Then we have

$$
v(t)=\frac{1}{\lambda}\left(\cos \theta(t) \partial_{x}+\sin \theta(t) \partial_{y}\right),
$$

and thus

$$
\begin{aligned}
\frac{\mathrm{D} v}{\mathrm{~d} t}=- & \frac{\dot{\lambda}}{\lambda^{2}}\left(\cos \theta \partial_{x}+\sin \theta \partial_{y}\right)+\frac{\dot{\theta}}{\lambda}\left(-\sin \theta \partial_{x}+\cos \theta \partial_{y}\right) \\
& +\frac{1}{\lambda}\left(\cos \theta\left(\dot{x} \nabla_{\partial_{x}} \partial_{x}+\dot{y} \nabla_{\partial_{y}} \partial_{x}\right)+\sin \theta\left(\dot{x} \nabla_{\partial_{x}} \partial_{y}+\dot{y} \nabla_{\partial_{y}} \partial_{y}\right)\right),
\end{aligned}
$$

where the dot denotes the derivation with respect to $t$. Since $\dot{\lambda}=\dot{x} \lambda_{x}+\dot{y} \lambda_{y}$, $\nabla_{\partial_{x}} \partial_{x}=\frac{\lambda_{x}}{\lambda} \partial_{x}-\frac{\lambda_{y}}{\lambda} \partial_{y}, \nabla_{\partial_{y}} \partial_{y}=-\frac{\lambda_{x}}{\lambda} \partial_{x}+\frac{\lambda_{y}}{\lambda} \partial_{y}$ and $\nabla_{\partial_{x}} \partial_{y}=\nabla_{\partial_{y}} \partial_{x}=\frac{\lambda_{y}}{\lambda} \partial_{x}+\frac{\lambda_{x}}{\lambda} \partial_{y}$, we get

$$
\frac{\mathrm{D} v}{\mathrm{~d} t}=\frac{1}{\lambda^{2}}\left(\lambda \dot{\theta}+\dot{y} \lambda_{x}-\dot{x} \lambda_{y}\right)\left(\cos \theta \partial_{y}-\sin \theta \partial_{x}\right) .
$$

Thus

$$
\|V\|_{(p, v)}^{2}=\lambda^{2}\left(\dot{x}^{2}+\dot{y}^{2}\right)+\frac{1}{\lambda^{2}}\left(\lambda \dot{\theta}+\dot{y} \lambda_{x}-\dot{x} \lambda_{y}\right)^{2} .
$$

Setting $z=\theta$ on the universal covering, we get the following expression for the metric of $\widetilde{U D}$ :

$$
\mathrm{d} s^{2}=\lambda^{2}\left(\mathrm{~d} x^{2}+\mathrm{d} y^{2}\right)+\left(-\frac{\lambda y}{\lambda} \mathrm{d} x+\frac{\lambda x}{\lambda} \mathrm{d} y+\mathrm{d} z\right)^{2} .
$$

We now choose $D=\mathbb{S}^{2} \backslash\{\infty\}$ with the metric of constant curvature 4 (i.e., the metric of the round sphere of radius $\frac{1}{2}$ ) given by the stereographic projection, i.e.,

$$
\lambda=\frac{1}{1+x^{2}+y^{2}} .
$$

Then we get

$$
\mathrm{d} s^{2}=\lambda^{2}\left(\mathrm{~d} x^{2}+\mathrm{d} y^{2}\right)+(2 \lambda(y \mathrm{~d} x-x \mathrm{~d} y)+\mathrm{d} z)^{2} .
$$

More generally, $\mathbb{R}^{3}$ endowed with the metric

$$
\mathrm{d} s^{2}=\lambda^{2}\left(\mathrm{~d} x^{2}+\mathrm{d} y^{2}\right)+(\tau \lambda(y \mathrm{~d} x-x \mathrm{~d} y)+\mathrm{d} z)^{2}
$$

with

$$
\lambda=\frac{1}{1+\frac{\kappa}{4}\left(x^{2}+y^{2}\right)}
$$

is the universal cover of a homogeneous manifold $\mathbb{E}$ of bundle curvature $\tau$ and of base curvature $\kappa>0$ minus the fiber corresponding to the point $\infty \in \mathbb{S}^{2}$. The fibers are 
given by $\left\{x=x_{0}, y=y_{0}\right\}$ in these coordinates. The canonical frame is $\left(E_{1}, E_{2}, E_{3}\right)$ with

$$
\begin{aligned}
& E_{1}=\lambda^{-1}\left(\cos (\sigma z) \partial_{x}+\sin (\sigma z) \partial_{y}\right)+\tau(x \sin (\sigma z)-y \cos (\sigma z)) \partial_{z}, \\
& E_{2}=\lambda^{-1}\left(-\sin (\sigma z) \partial_{x}+\cos (\sigma z) \partial_{y}\right)+\tau(x \cos (\sigma z)+y \sin (\sigma z)) \partial_{z}, \\
& E_{3}=\partial_{z}
\end{aligned}
$$

with

$$
\sigma=\frac{\kappa}{2 \tau}
$$

which satisfy

$$
\left[E_{1}, E_{2}\right]=2 \tau E_{3}, \quad\left[E_{2}, E_{3}\right]=\frac{\kappa}{2 \tau} E_{1}, \quad\left[E_{3}, E_{1}\right]=\frac{\kappa}{2 \tau} E_{2} .
$$

This frame is defined on the open set $\mathbb{E}^{\prime}$ which is $\mathbb{E}$ minus the fiber corresponding to the point $\infty \in \mathbb{S}^{2}$.

The Berger spheres in the strict sense are the manifolds such that $\kappa=4$.

2.3. The manifolds with the isometry group of the Heisenberg group $\mathrm{Nil}_{3}$. They occur when $\tau \neq 0$ and $\kappa=0$; they are fibrations over the Euclidean plane.

The Heisenberg group is the Lie group

$$
\mathrm{Nil}_{3}=\left\{\left(\begin{array}{ccc}
1 & a & c \\
0 & 1 & b \\
0 & 0 & 1
\end{array}\right) ;(a, b, c) \in \mathbb{R}^{3}\right\}
$$

endowed with a left invariant metric.

It is useful to use exponential coordinates. In this model, the Heisenberg space $\mathrm{Nil}_{3}$ is $\mathbb{R}^{3}$ endowed with the following metric:

$$
\mathrm{d} s^{2}=\mathrm{d} x^{2}+\mathrm{d} y^{2}+(\tau(y \mathrm{~d} x-x \mathrm{~d} y)+\mathrm{d} z)^{2} .
$$

The fibers are given by $\left\{x=x_{0}, y=y_{0}\right\}$ in these coordinates.

The canonical frame is $\left(E_{1}, E_{2}, E_{3}\right)$ with

$$
E_{1}=\partial_{x}-\tau y \partial_{z}, \quad E_{2}=\partial_{y}+\tau x \partial_{z}, \quad E_{3}=\partial_{z},
$$

which satisfy

$$
\left[E_{1}, E_{2}\right]=2 \tau E_{3}, \quad\left[E_{2}, E_{3}\right]=0, \quad\left[E_{3}, E_{1}\right]=0 .
$$

The reader can refer to [FMP99] (where $\tau=\frac{1}{2}$ ). 
2.4. The manifolds with the isometry group of $\widetilde{\operatorname{PSL}_{2}(\mathbb{R})}$. They occur when $\tau \neq 0$ and $\kappa<0$; they are fibrations over hyperbolic planes.

The Lie group $\widetilde{\mathrm{PL}_{2}(\mathbb{R})}$ with its standard metric can be identified with the universal covering of the unitary tangent bundle to the hyperbolic plane $\mathrm{UH}^{2}$ equipped with its canonical metric. Indeed, the group $\mathrm{PSL}_{2}(\mathbb{R})$ acts transitively on $\mathrm{UH}^{2}$, and the stabilizer of any point in $\mathrm{UH}^{2}$ is trivial. The unitary tangent bundle $\mathrm{UH}^{2}$ can be endowed with the metric induced by the standard metric on the tangent bundle $\mathrm{TH}^{2}$. The reader can refer to [Sco83]. We will give an expression of this metric.

Let $(x, y) \mapsto \varphi(x, y)$ be a conformal parametrization of $\mathbb{H}^{2}$ and let $\lambda$ be the conformal factor, i.e., the metric of $\mathbb{H}^{2}$ is given by $\lambda^{2}\left(\mathrm{~d} x^{2}+\mathrm{d} y^{2}\right)$. Then, proceeding as in Section 2.2, we obtain that a metric on $\widehat{\mathrm{PSL}_{2}(\mathbb{R})}$ is

$$
\mathrm{d} s^{2}=\lambda^{2}\left(\mathrm{~d} x^{2}+\mathrm{d} y^{2}\right)+\left(-\frac{\lambda y}{\lambda} \mathrm{d} x+\frac{\lambda_{x}}{\lambda} \mathrm{d} y+\mathrm{d} z\right)^{2} .
$$

This metric defines a homogeneous manifold with $\kappa=-1$ and $\tau=-\frac{1}{2}$.

More generally, we can take the Poincaré disk model for the hyperbolic plane of constant curvature $\kappa<0$. The manifold $\mathbb{D}^{2}\left(\frac{2}{\sqrt{-\kappa}}\right) \times \mathbb{R}$, where $\mathbb{D}^{2}(\rho)=\{(x, y) \in$ $\left.\mathbb{R}^{2} ; x^{2}+y^{2}<\rho^{2}\right\}$, endowed with the metric

$$
\mathrm{d} s^{2}=\lambda^{2}\left(\mathrm{~d} x^{2}+\mathrm{d} y^{2}\right)+(\tau \lambda(y \mathrm{~d} x-x \mathrm{~d} y)+\mathrm{d} z)^{2}
$$

with

$$
\lambda=\frac{1}{1+\frac{\kappa}{4}\left(x^{2}+y^{2}\right)},
$$

is a homogeneous manifold of bundle curvature $\tau$ and of base curvature $\kappa<0$. The fibers are given by $\left\{x=x_{0}, y=y_{0}\right\}$ in these coordinates. The canonical frame is $\left(E_{1}, E_{2}, E_{3}\right)$ with

$$
\begin{aligned}
& E_{1}=\lambda^{-1}\left(\cos (\sigma z) \partial_{x}+\sin (\sigma z) \partial_{y}\right)+\tau(x \sin (\sigma z)-y \cos (\sigma z)) \partial_{z}, \\
& E_{2}=\lambda^{-1}\left(-\sin (\sigma z) \partial_{x}+\cos (\sigma z) \partial_{y}\right)+\tau(x \cos (\sigma z)+y \sin (\sigma z)) \partial_{z}, \\
& E_{3}=\partial_{z}
\end{aligned}
$$

with

$$
\sigma=\frac{\kappa}{2 \tau}
$$

which satisfy

$$
\left[E_{1}, E_{2}\right]=2 \tau E_{3}, \quad\left[E_{2}, E_{3}\right]=\frac{\kappa}{2 \tau} E_{1}, \quad\left[E_{3}, E_{1}\right]=\frac{\kappa}{2 \tau} E_{2} .
$$




\section{Preliminaries}

3.1. The compatibility equations for surfaces in 3-dimensional homogeneous manifolds. We consider a 3-dimensional homogeneous manifold $\mathbb{E}$ with an isometry group of dimension 4, of bundle curvature $\tau$ and of base curvature $\kappa$. Let $\bar{R}$ be the Riemann curvature tensor of $\mathbb{E}$. Let $\mathcal{V}$ be an oriented surface in $\mathbb{E}, \nabla$ the Riemannian connection of $\mathcal{V}, \mathrm{J}$ the rotation of angle $\frac{\pi}{2}$ on TV,$N$ the unit normal to $\mathcal{V}$ and $\mathrm{S}$ the shape operator of $\mathcal{V}$.

Proposition 3.1. For $X, Y, Z, W \in \mathfrak{X}(\mathcal{V})$ we have

$$
\begin{gathered}
\langle\overline{\mathrm{R}}(X, Y) Z, W\rangle=\left(\kappa-3 \tau^{2}\right)\left\langle\mathrm{R}_{0}(X, Y) Z, W\right\rangle+\left(\kappa-4 \tau^{2}\right)\left\langle\mathrm{R}_{1}(T ; X, Y) Z, W\right\rangle, \\
\overline{\mathrm{R}}(X, Y) N=\left(\kappa-4 \tau^{2}\right) v(\langle Y, T\rangle X-\langle X, T\rangle Y),
\end{gathered}
$$

where

$$
v=\langle N, \xi\rangle,
$$

$T$ is the projection of $\xi$ on $\mathrm{T} \mathcal{V}$, i.e.,

$$
T=\xi-v N,
$$

and $\mathrm{R}_{0}$ and $\mathrm{R}_{1}$ are as in Proposition 2.1.

Proof. This is a consequence of Proposition 2.1, using the fact that $X, Y$ and $Z$ are tangent to the surface and $N$ is normal to the surface.

Corollary 3.2. The Gauss and Codazzi equations in $\mathbb{E}$ are

$$
\begin{gathered}
K=\operatorname{det} \mathrm{S}+\tau^{2}+\left(\kappa-4 \tau^{2}\right) \nu^{2}, \\
\nabla_{X} \mathrm{~S} Y-\nabla_{Y} \mathrm{~S} X-\mathrm{S}[X, Y]=\left(\kappa-4 \tau^{2}\right) v(\langle Y, T\rangle X-\langle X, T\rangle Y),
\end{gathered}
$$

where $K$ is the Gauss curvature of $\mathcal{V}$.

Proposition 3.3. For $X \in \mathfrak{X}(\mathcal{V})$ we have

$$
\nabla_{X} T=v(\mathbf{S} X-\tau \mathbf{J} X), \quad \mathrm{d} v(X)+\langle\mathbf{S} X-\tau \mathbf{J} X, T\rangle=0 .
$$

Proof. On the one hand we have

$$
\begin{aligned}
\bar{\nabla}_{X} \xi & =\bar{\nabla}_{X}(T+v N) \\
& =\bar{\nabla}_{X} T+\mathrm{d} v(X) N+v \bar{\nabla}_{X} N \\
& =\nabla_{X} T+\langle\mathrm{S} X, T\rangle N+\mathrm{d} v(X) N-v \mathrm{~S} X .
\end{aligned}
$$


On the other hand we have

$$
\begin{aligned}
\bar{\nabla}_{X} \xi & =\tau X \times \xi \\
& =\tau X \times(T+\nu N) \\
& =\tau(\langle\mathrm{J} X, T\rangle N-v \mathrm{~J} X) .
\end{aligned}
$$

We conclude taking the tangential and normal parts in both expressions.

3.2. Moving frames. In this section we introduce some material about the technique of moving frames.

Let $\mathcal{V}$ be a Riemannian manifold of dimension $n, \nabla$ its Levi-Civita connection, and $\mathrm{R}$ the Riemannian curvature tensor. Let $\mathrm{S}$ be a field of symmetric operators $\mathrm{S}_{y}: \mathrm{T}_{y} \mathcal{V} \rightarrow \mathrm{T}_{y} \mathcal{V}$. Let $\left(e_{1}, \ldots, e_{n}\right)$ be a local orthonormal frame on $\mathcal{V}$ and $\left(\omega^{1}, \ldots, \omega^{n}\right)$ the dual basis of $\left(e_{1}, \ldots, e_{n}\right)$, i.e.,

$$
\omega^{i}\left(e_{k}\right)=\delta_{k}^{i} .
$$

We also set

$$
\omega^{n+1}=0 .
$$

We define the forms $\omega_{j}^{i}, \omega_{j}^{n+1}, \omega_{n+1}^{i}$ and $\omega_{n+1}^{n+1}$ on $\mathcal{V}$ by

$$
\begin{gathered}
\omega_{j}^{i}\left(e_{k}\right)=\left\langle\nabla_{e_{k}} e_{j}, e_{i}\right\rangle, \quad \omega_{j}^{n+1}\left(e_{k}\right)=\left\langle\mathrm{S} e_{k}, e_{j}\right\rangle, \\
\omega_{n+1}^{j}=-\omega_{j}^{n+1}, \quad \omega_{n+1}^{n+1}=0 .
\end{gathered}
$$

Then we have

$$
\nabla_{e_{k}} e_{j}=\sum_{i} \omega_{j}^{i}\left(e_{k}\right) e_{i}, \quad \mathrm{~S} e_{k}=\sum_{j} \omega_{j}^{n+1}\left(e_{k}\right) e_{j} .
$$

Finally we set $R_{k l j}^{i}=\left\langle\mathrm{R}\left(e_{k}, e_{l}\right) e_{j}, e_{i}\right\rangle$.

Proposition 3.4. We have the following formulas:

$$
\begin{gathered}
\mathrm{d} \omega^{i}+\sum_{p} \omega_{p}^{i} \wedge \omega^{p}=0 \\
\sum_{p} \omega_{p}^{n+1} \wedge \omega^{p}=0 \\
\mathrm{~d} \omega_{j}^{i}+\sum_{p} \omega_{p}^{i} \wedge \omega_{j}^{p}=-\frac{1}{2} \sum_{k} \sum_{l} R_{k l j}^{i} \omega^{k} \wedge \omega^{l} \\
\mathrm{~d} \omega_{j}^{n+1}+\sum_{p} \omega_{p}^{n+1} \wedge \omega_{j}^{p}=\frac{1}{2} \sum_{k} \sum_{l}\left\langle\nabla_{e_{k}} \mathrm{~S} e_{l}-\nabla_{e_{l}} \mathrm{~S} e_{k}-\mathrm{S}\left[e_{k}, e_{l}\right], e_{j}\right\rangle \omega^{k} \wedge \omega^{l}
\end{gathered}
$$


For a proof of these classical formulas, the reader can refer to [Dan04], Proposition 2.4 .

3.3. Some facts about hypersurfaces. In this section we consider an orientable hypersurface $\mathcal{V}$ of an $(n+1)$-dimensional Riemannian manifold $\overline{\mathcal{V}}$. Let $\left(e_{1}, \ldots, e_{n}\right)$ be a local orthonormal frame on $\mathcal{V}, e_{n+1}$ the normal to $\mathcal{V}$, and $\left(E_{1}, \ldots, E_{n+1}\right)$ a local orthonormal frame on $\overline{\mathcal{V}}$. We denote by $\nabla$ and $\bar{\nabla}$ the Riemannian connections on $\mathcal{V}$ and $\overline{\mathcal{V}}$ respectively, and by $\mathrm{S}$ the shape operator of $\mathcal{V}$ (with respect to the normal $\left.e_{n+1}\right)$. We define the forms $\omega^{\alpha}, \omega_{\beta}^{\alpha}$ on $\mathcal{V}$ as in Section 3.2. Then we have

$$
\bar{\nabla}_{e_{k}} e_{\beta}=\sum_{\gamma} \omega_{\beta}^{\gamma}\left(e_{k}\right) e_{\gamma}
$$

Let $A \in \mathrm{SO}_{n+1}(\mathbb{R})$ be the matrix whose columns are the coordinates of the $e_{\beta}$ in the frame $\left(E_{\alpha}\right)$, namely $A_{\beta}^{\alpha}=\left\langle e_{\beta}, E_{\alpha}\right\rangle$. Let $\Omega=\left(\omega_{\beta}^{\alpha}\right) \in \mathcal{M}_{n+1}(\mathbb{R})$.

Lemma 3.5. The matrix A satisfies the following equation:

$$
A^{-1} \mathrm{~d} A=\Omega+L(A)
$$

with

$$
L(A)_{\beta}^{\alpha}=\sum_{k}\left(\sum_{\gamma, \delta, \varepsilon} A_{\alpha}^{\varepsilon} A_{k}^{\gamma} A_{\beta}^{\delta} \bar{\Gamma}_{\gamma \varepsilon}^{\delta}\right) \omega^{k}
$$

where the $\bar{\Gamma}_{\gamma \varepsilon}^{\delta}$ are the Christoffel symbols of the frame $\left(E_{\alpha}\right)$.

Proof. We have

$$
e_{\beta}=\sum_{\alpha} A_{\beta}^{\alpha} E_{\alpha}
$$

Then, on the one hand we have

$$
\begin{aligned}
\bar{\nabla}_{e_{k}} e_{\beta} & =\sum_{\delta} \mathrm{d} A_{\beta}^{\delta}\left(e_{k}\right) E_{\delta}+\sum_{\delta} A_{\beta}^{\delta} \bar{\nabla}_{e_{k}} E_{\delta} \\
& =\sum_{\varepsilon} \mathrm{d} A_{\beta}^{\varepsilon}\left(e_{k}\right) E_{\delta}+\sum_{\gamma} \sum_{\delta} \sum_{\varepsilon} A_{\beta}^{\delta} A_{k}^{\gamma} \bar{\Gamma}_{\gamma \delta}^{\varepsilon} E_{\varepsilon},
\end{aligned}
$$

and on the other hand we have

$$
\bar{\nabla}_{e_{k}} e_{\beta}=\sum_{\gamma} \sum_{\varepsilon} \omega_{\beta}^{\gamma}\left(e_{k}\right) A_{\gamma}^{\varepsilon} E_{\varepsilon} .
$$


Identifying the coefficients we get

$$
\begin{aligned}
\mathrm{d} A_{\beta}^{\varepsilon}\left(e_{k}\right) & =-\sum_{\gamma} \sum_{\delta} A_{\beta}^{\delta} A_{k}^{\gamma} \bar{\Gamma}_{\gamma \delta}^{\varepsilon}+\sum_{\gamma} \omega_{\beta}^{\gamma}\left(e_{k}\right) A_{\gamma}^{\varepsilon} \\
& =\sum_{\gamma} \sum_{\delta} A_{\beta}^{\delta} A_{k}^{\gamma} \bar{\Gamma}_{\gamma \varepsilon}^{\delta}+\sum_{\gamma} \omega_{\beta}^{\gamma}\left(e_{k}\right) A_{\gamma}^{\varepsilon}
\end{aligned}
$$

since the frame $\left(E_{\alpha}\right)$ is orthonormal.

We conclude using the fact that $A^{-1}$ is the transpose of $A$.

\section{Isometric immersions of surfaces into 3-dimensional homogeneous manifolds}

We consider a simply connected oriented Riemannian manifold $\mathcal{V}$ of dimension 2 . Let $\mathrm{d} s^{2}$ be the metric on $\mathcal{V}$ (we will also denote it by $\langle\cdot, \cdot\rangle$ ), $\nabla$ the Riemannian connection of $\mathcal{V}, \mathrm{R}$ its Riemann curvature tensor, $K$ its Gauss curvature, and $\mathrm{J}$ the rotation of angle $\frac{\pi}{2}$ on $T \mathcal{V}$. Let $\mathrm{S}$ be a field of symmetric operators $\mathrm{S}_{y}: \mathrm{T}_{y} \mathcal{V} \rightarrow \mathrm{T}_{y} \mathcal{V}, T$ a vector field on $\mathcal{V}$ such that $\|T\| \leqslant 1$ and $v$ a smooth function on $\mathcal{V}$ such that $v^{2} \leqslant 1$.

The compatibility equations for surfaces in 3-dimensional homogeneous manifolds with 4-dimensional isometry group established in Section 3.1 suggest to introduce the following definition.

Definition 4.1. Let $\mathbb{E}$ be a 3-dimensional homogeneous manifold with a 4-dimensional isometry group. Let $\kappa$ be its base curvature and $\tau$ its bundle curvature. We say that $\left(\mathrm{d} s^{2}, \mathrm{~S}, T, v\right)$ satisfies the compatibility equations for $\mathbb{E}$ if

$$
\|T\|^{2}+v^{2}=1
$$

and, for all $X, Y, Z \in \mathfrak{X}(\mathcal{V})$,

$$
\begin{gathered}
K=\operatorname{det} \mathrm{S}+\tau^{2}+\left(\kappa-4 \tau^{2}\right) \nu^{2}, \\
\nabla_{X} \mathrm{~S} Y-\nabla_{Y} \mathrm{~S} X-\mathrm{S}[X, Y]=\left(\kappa-4 \tau^{2}\right) \nu(\langle Y, T\rangle X-\langle X, T\rangle Y), \\
\nabla_{X} T=v(\mathrm{~S} X-\tau \mathrm{J} X), \\
\mathrm{d} v(X)+\langle\mathrm{S} X-\tau \mathrm{J} X, T\rangle=0 .
\end{gathered}
$$

Remark 4.2. We notice that (10) implies (11) except when $v=0$ (by differentiating the identity $\langle T, T\rangle+v^{2}=1$ with respect to $\left.X\right)$. 
Theorem 4.3. Let $\mathcal{V}$ be a simply connected oriented Riemannian manifold of dimension $2, \mathrm{~d} s^{2}$ its metric and $\nabla$ its Riemannian connection. Let $\mathrm{S}$ be a field of symmetric operators $S_{y}: T_{y} \mathcal{V} \rightarrow T_{y} \mathcal{V}, T$ a vector field on $\mathcal{V}$ and $v$ a smooth function on $\mathcal{V}$ such that $\|T\|^{2}+v^{2}=1$.

Let $\mathbb{E}$ be a 3-dimensional homogeneous manifold with a 4-dimensional isometry group and $\xi$ its vertical vector field. Let $\kappa$ be its base curvature and $\tau$ its bundle curvature. Then there exists an isometric immersion $f: \mathcal{V} \rightarrow \mathbb{E}$ such that the shape operator with respect to the normal $N$ associated to $f$ is

$$
\mathrm{d} f \circ \mathrm{S} \circ \mathrm{d} f^{-1}
$$

and such that

$$
\xi=\mathrm{d} f(T)+v N
$$

if and only if $\left(\mathrm{d} s^{2}, \mathrm{~S}, T, v\right)$ satisfies the compatibility equations for $\mathbb{E}$. In this case, the immersion is unique up to a global isometry of $\mathbb{E}$ preserving the orientations of both the fibers and the base of the fibration.

The fact that the compatibility equations are necessary was proved in Section 3.1. To prove that they are sufficient, we consider a local orthonormal frame $\left(e_{1}, e_{2}\right)$ on $\mathcal{V}$ and the forms $\omega^{i}, \omega^{3}, \omega_{j}^{i}, \omega_{j}^{3}, \omega_{3}^{i}$ and $\omega_{3}^{3}$ as in Section 3.2 (with $n=2$ ).

From now on we assume that $\tau \neq 0$ since the case $\tau=0$ was treated in [Dan04].

We denote by $\left(E_{1}, E_{2}, E_{3}\right)$ the canonical frame of $\mathbb{E}$ (see Section 2.1); in particular we have $E_{3}=\xi$. We denote by $\mathbb{E}^{\prime}$ the open set where the canonical frame is defined (in particular we have $\mathbb{E}^{\prime}=\mathbb{E}$ when $\kappa=0$ or $\kappa<0$; see Sections 2.2, 2.3 and 2.4).

We set

$$
T^{k}=\left\langle T, e_{k}\right\rangle, \quad T^{3}=v .
$$

We define the one-form $\eta$ on $\mathcal{V}$ by

$$
\eta(X)=\langle T, X\rangle .
$$

In the frame $\left(e_{1}, e_{2}\right)$ we have $\eta=\sum_{k} T^{k} \omega^{k}$. We define the following matrix of one-forms:

$$
\Omega=\left(\omega_{\beta}^{\alpha}\right) \in \mathcal{M}_{3}(\mathbb{R}) .
$$

For a point $y \in \mathcal{V}$ where $\left(e_{1}, e_{2}\right)$ is defined, let $\mathcal{Z}(y)$ be the set of matrices $Z \in \mathrm{SO}_{3}(\mathbb{R})$ such that the coefficients of the last line of $Z$ are the $T^{\beta}(y)$. It is diffeomorphic to the circle $\mathbb{S}^{1}$. For $Z \in \mathcal{Z}(y)$, we set

$$
L(Z)_{\beta}^{\alpha}=\sum_{k}\left(\sum_{\gamma, \delta, \varepsilon} Z_{\alpha}^{\varepsilon} Z_{k}^{\gamma} Z_{\beta}^{\delta} \bar{\Gamma}_{\gamma \varepsilon}^{\delta}\right) \omega^{k}
$$

where the $\bar{\Gamma}_{\gamma \varepsilon}^{\delta}$ are the Christoffel symbols of the frame $\left(E_{\alpha}\right)$ (see Section 3.3). This defines an antisymmetric matrix of 1 -forms. 
We also set $\sigma=\frac{\kappa}{2 \tau}$.

From now on we assume that the hypotheses of Theorem 4.3 are satisfied. We first prove some technical lemmas that are consequences of the compatibility equations.

Lemma 4.4. We have

$$
\mathrm{d} \eta=-2 \tau \nu \omega^{1} \wedge \omega^{2} .
$$

Proof. By (10) we have $\mathrm{d} \eta(X, Y)=\left\langle\nabla_{X} T, Y\right\rangle-\left\langle\nabla_{Y} T, X\right\rangle=2 \tau \nu\langle X, \mathrm{~J} Y\rangle$. Thus $\mathrm{d} \eta\left(e_{1}, e_{2}\right)=-2 \tau \nu$.

Lemma 4.5. We have

$$
\begin{gathered}
\mathrm{d} T^{1}=\sum_{\gamma} T^{\gamma} \omega_{1}^{\gamma}+\tau T^{3} \omega^{2}, \\
\mathrm{~d} T^{2}=\sum_{\gamma} T^{\gamma} \omega_{2}^{\gamma}-\tau T^{3} \omega^{1}, \\
\mathrm{~d} T^{3}=\sum_{\gamma} T^{\gamma} \omega_{3}^{\gamma}-\tau T^{1} \omega^{2}+\tau T^{2} \omega^{1} .
\end{gathered}
$$

Proof. The first two identities are a consequence of condition (10) and the last one of condition (11).

Lemma 4.6. We have

$$
\begin{array}{r}
\mathrm{d} \Omega+\Omega \wedge \Omega=\left(\begin{array}{ccc}
0 & \tau^{2} & 0 \\
-\tau^{2} & 0 & 0 \\
0 & 0 & 0
\end{array}\right) \omega^{1} \wedge \omega^{2} \\
+\left(\kappa-4 \tau^{2}\right) T^{3}\left(\begin{array}{ccc}
0 & T^{3} & -T^{2} \\
-T^{3} & 0 & T^{1} \\
T^{2} & -T^{1} & 0
\end{array}\right) \omega^{1} \wedge \omega^{2} .
\end{array}
$$

Proof. We set $\Psi=\mathrm{d} \Omega+\Omega \wedge \Omega$ and $R_{k l j}^{i}=\left\langle\mathrm{R}\left(e_{k}, e_{l}\right) e_{j}, e_{i}\right\rangle$. By Proposition 3.4 we have

$$
\Psi_{j}^{i}=-\frac{1}{2} \sum_{k} \sum_{l} R_{k l j}^{i} \omega^{k} \wedge \omega^{l}+\omega_{3}^{i} \wedge \omega_{j}^{3}
$$

and by the Gauss equation (8) we have $R_{k l j}^{i}=\bar{R}_{k l j}^{i}+\omega_{j}^{3} \wedge \omega_{i}^{3}\left(e_{k}, e_{l}\right)$ with $\bar{R}_{k l j}^{i}=\left(\kappa-3 \tau^{2}\right)\left(\delta_{j}^{k} \delta_{i}^{l}-\delta_{j}^{l} \delta_{i}^{k}\right)+\left(\kappa-4 \tau^{2}\right)\left(T^{l} T^{j} \delta_{i}^{k}+T^{k} T^{i} \delta_{j}^{l}-T^{l} T^{i} \delta_{j}^{k}-T^{k} T^{j} \delta_{i}^{l}\right)$.

Thus we get

$$
\Psi_{j}^{i}=\left(\kappa-3 \tau^{2}\right) \omega^{i} \wedge \omega^{j}+\left(\kappa-4 \tau^{2}\right)\left(T^{i} \omega^{j}-T^{j} \omega^{i}\right) \wedge \eta .
$$


In the same way, by Proposition 3.4 we have

$$
\Psi_{j}^{3}=\frac{1}{2} \sum_{k} \sum_{l}\left\langle\nabla_{e_{k}} \mathrm{~S} e_{l}-\nabla_{e_{l}} \mathrm{~S} e_{k}-\mathrm{S}\left[e_{k}, e_{l}\right], e_{j}\right\rangle \omega^{k} \wedge \omega^{l}
$$

and by the Codazzi equation (9) we have

$$
\left\langle\nabla_{e_{k}} \mathrm{~S} e_{l}-\nabla_{e_{l}} \mathrm{~S} e_{k}-\mathrm{S}\left[e_{k}, e_{l}\right], e_{j}\right\rangle=\left(\kappa-4 \tau^{2}\right) T^{3}\left(T^{l} \delta_{j}^{k}-T^{k} \delta_{j}^{l}\right)
$$

Thus we get

$$
\Psi_{j}^{3}=\left(\kappa-4 \tau^{2}\right) T^{3} \omega^{j} \wedge \eta
$$

Hence we have

$$
\begin{aligned}
\Psi=(\kappa & \left.-3 \tau^{2}\right)\left(\begin{array}{ccc}
0 & 1 & 0 \\
-1 & 0 & 0 \\
0 & 0 & 0
\end{array}\right) \omega^{1} \wedge \omega^{2} \\
& +\left(\kappa-4 \tau^{2}\right)\left(\begin{array}{ccc}
0 & -T^{2} & -T^{3} \\
T^{2} & 0 & 0 \\
T^{3} & 0 & 0
\end{array}\right) \omega^{1} \wedge \eta \\
& +\left(\kappa-4 \tau^{2}\right)\left(\begin{array}{ccc}
0 & T^{1} & -0 \\
-T^{1} & 0 & -T^{3} \\
0 & T^{3} & 0
\end{array}\right) \omega^{2} \wedge \eta .
\end{aligned}
$$

We conclude using that $\omega^{1} \wedge \eta=T^{2} \omega^{1} \wedge \omega^{2}, \omega^{2} \wedge \eta=-T^{1} \omega^{1} \wedge \omega^{2}$ and $\left(T^{1}\right)^{2}+$ $\left(T^{2}\right)^{2}+\left(T^{3}\right)^{2}=1$.

Lemma 4.7. We have

$$
\begin{aligned}
L(Z)=(2 \tau-\sigma) & \left(\begin{array}{ccc}
0 & -T^{3} & T^{2} \\
T^{3} & 0 & -T^{1} \\
-T^{2} & T^{1} & 0
\end{array}\right) \eta \\
& +\left(\begin{array}{ccc}
0 & 0 & 0 \\
0 & 0 & \tau \\
0 & -\tau & 0
\end{array}\right) \omega^{1}+\left(\begin{array}{ccc}
0 & 0 & -\tau \\
0 & 0 & 0 \\
\tau & 0 & 0
\end{array}\right) \omega^{2} .
\end{aligned}
$$


Proof. We compute that

$$
\begin{aligned}
L(Z)_{\beta}^{\alpha}= & \sum_{k}\left(\sum_{\gamma} \sum_{\delta} \sum_{\varepsilon} Z_{\alpha}^{\varepsilon} Z_{k}^{\gamma} Z_{\beta}^{\delta} \bar{\Gamma}_{\gamma \varepsilon}^{\delta}\right) \omega^{k} \\
= & \sum_{k}\left(\tau\left(Z_{\alpha}^{2} Z_{k}^{1} Z_{\beta}^{3}+Z_{\alpha}^{3} Z_{k}^{2} Z_{\beta}^{1}-Z_{\alpha}^{1} Z_{k}^{2} Z_{\beta}^{3}-Z_{\alpha}^{3} Z_{k}^{1} Z_{\beta}^{2}\right)\right. \\
& \left.\quad+(\tau-\sigma)\left(Z_{\alpha}^{2} Z_{k}^{3} Z_{\beta}^{1}-Z_{\alpha}^{1} Z_{k}^{3} Z_{\beta}^{2}\right)\right) \omega^{k} \\
= & \sum_{k}\left(\tau T^{\beta}\left(Z_{k}^{1} Z_{\alpha}^{2}-Z_{\alpha}^{1} Z_{k}^{2}\right)+\tau T^{\alpha}\left(Z_{\beta}^{1} Z_{k}^{2}-Z_{k}^{1} Z_{\beta}^{2}\right)\right. \\
& \left.\quad+(\tau-\sigma) T^{k}\left(Z_{\beta}^{1} Z_{\alpha}^{2}-Z_{\alpha}^{1} Z_{\beta}^{2}\right)\right) \omega^{k} .
\end{aligned}
$$

Moreover the matrix $Z$ lies in $\mathrm{SO}_{3}(\mathbb{R})$, so it is equal to its comatrix. Using this fact we compute that

$$
\begin{aligned}
& L(Z)_{2}^{1}=-(2 \tau-\sigma) T^{3}\left(T^{1} \omega^{1}+T^{2} \omega^{2}\right), \\
& L(Z)_{3}^{1}=(2 \tau-\sigma) T^{1} T^{2} \omega^{1}+(2 \tau-\sigma)\left(T^{2}\right)^{2} \omega^{2}-\tau \omega^{2}, \\
& L(Z)_{3}^{2}=-(2 \tau-\sigma)\left(T^{1}\right)^{2} \omega^{1}-(2 \tau-\sigma) T^{1} T^{2} \omega^{2}+\tau \omega^{1},
\end{aligned}
$$

which proves the lemma.

Lemma 4.8. We have

$$
\begin{aligned}
L \wedge L= & \tau(2 \tau-\sigma) T^{3}\left(\begin{array}{ccc}
0 & -T^{3} & T^{2} \\
T^{3} & 0 & -T^{1} \\
-T^{2} & T^{1} & 0
\end{array}\right) \omega^{1} \wedge \omega^{2} \\
& +\tau(\tau-\sigma)\left(\begin{array}{ccc}
0 & 1 & 0 \\
-1 & 0 & 0 \\
0 & 0 & 0
\end{array}\right) \omega^{1} \wedge \omega^{2} .
\end{aligned}
$$

Proof. We compute that

$$
\begin{aligned}
& L \wedge L=\tau(2 \tau-\sigma)\left(\begin{array}{ccc}
0 & T^{1} & 0 \\
-T^{1} & 0 & -T^{3} \\
0 & T^{3} & 0
\end{array}\right) \eta \wedge \omega^{2} \\
& \quad+\tau(2 \tau-\sigma)\left(\begin{array}{ccc}
0 & -T^{2} & -T^{3} \\
T^{2} & 0 & 0 \\
T^{3} & 0 & 0
\end{array}\right) \eta \wedge \omega^{1}+\tau^{2}\left(\begin{array}{ccc}
0 & -1 & 0 \\
1 & 0 & 0 \\
0 & 0 & 0
\end{array}\right) \omega^{1} \wedge \omega^{2}
\end{aligned}
$$

We conclude using that $\left(T^{1}\right)^{2}+\left(T^{2}\right)^{2}+\left(T^{3}\right)^{2}=1$. 
Lemma 4.9. We have

$$
\begin{aligned}
L \wedge \Omega+\Omega \wedge L=( & 2 \tau-\sigma) \eta \wedge\left(\begin{array}{ccc}
0 & -\mathrm{d} T^{3} & \mathrm{~d} T^{2} \\
\mathrm{~d} T^{3} & 0 & -\mathrm{d} T^{1} \\
-\mathrm{d} T^{2} & \mathrm{~d} T^{1} & 0
\end{array}\right) \\
& +\tau(2 \tau-\sigma) T^{3}\left(\begin{array}{ccc}
0 & T^{3} & -T^{2} \\
-T^{3} & 0 & T^{1} \\
T^{2} & -T^{1} & 0
\end{array}\right) \omega^{1} \wedge \omega^{2} \\
& +\tau(2 \tau-\sigma)\left(\begin{array}{ccc}
0 & -1 & 0 \\
1 & 0 & 0 \\
0 & 0 & 0
\end{array}\right) \omega^{1} \wedge \omega^{2} \\
& +\tau\left(\begin{array}{ccc}
0 & 0 & 0 \\
0 & 0 & -1 \\
0 & 1 & 0
\end{array}\right) \mathrm{d} \omega^{1}+\tau\left(\begin{array}{ccc}
0 & 0 & 1 \\
0 & 0 & 0 \\
-1 & 0 & 0
\end{array}\right) \mathrm{d} \omega^{2} .
\end{aligned}
$$

Proof. We compute that

$L \wedge \Omega+\Omega \wedge L=(2 \tau-\sigma) \eta \wedge M$

$$
+\tau \omega^{2} \wedge\left(\begin{array}{ccc}
0 & -\omega_{2}^{3} & 0 \\
-\omega_{3}^{2} & 0 & \omega_{1}^{2} \\
0 & \omega_{2}^{1} & 0
\end{array}\right)+\tau \omega^{1} \wedge\left(\begin{array}{ccc}
0 & \omega_{3}^{1} & -\omega_{2}^{1} \\
\omega_{1}^{3} & 0 & 0 \\
-\omega_{1}^{2} & 0 & 0
\end{array}\right)
$$

with

$$
M=\left(\begin{array}{ccc}
0 & T^{2} \omega_{2}^{3}-T^{1} \omega_{3}^{1} & -T^{3} \omega_{3}^{2}+T^{1} \omega_{2}^{1} \\
-T^{1} \omega_{1}^{3}+T^{2} \omega_{3}^{2} & 0 & T^{3} \omega_{3}^{1}-T^{2} \omega_{1}^{2} \\
T^{1} \omega_{1}^{2}-T^{3} \omega_{2}^{3} & -T^{2} \omega_{2}^{1}+T^{3} \omega_{1}^{3} & 0
\end{array}\right)
$$

We conclude using Lemma 4.5, formulas (4) and (5), and the fact that $\left(T^{1}\right)^{2}+$ $\left(T^{2}\right)^{2}+\left(T^{3}\right)^{2}=1$.

We now prove the following proposition.

Proposition 4.10. Assume that the compatibility equations for $\mathbb{E}$ are satisfied. Let $y_{0} \in \mathcal{V}$ and $A_{0} \in \mathcal{Z}\left(y_{0}\right)$. Then there exist a neighbourhood $U_{1}$ of $y_{0}$ in $\mathcal{V}$ and $a$ unique map $A: U_{1} \rightarrow \mathrm{SO}_{3}(\mathbb{R})$ such that

$$
\begin{gathered}
A^{-1} \mathrm{~d} A=\Omega+L(A), \\
A(y) \in \mathcal{Z}(y) \text { for all } y \in U_{1}, \\
A\left(y_{0}\right)=A_{0} .
\end{gathered}
$$


Proof. Let $U$ be a coordinate neighbourhood in $\mathcal{V}$. The set

$$
\mathscr{F}=\left\{(y, Z) \in U \times \mathrm{SO}_{3}(\mathbb{R}) ; Z \in \mathcal{Z}(y)\right\}
$$

is a manifold of dimension 3 , and

$$
\mathrm{T}_{(y, Z)} \mathcal{F}=\left\{(u, \zeta) \in \mathrm{T}_{y} U \oplus \mathrm{T}_{Z} \mathrm{SO}_{3}(\mathbb{R}) ; \zeta_{\beta}^{3}=\left(\mathrm{d} T^{\beta}\right)_{y}(u)\right\} .
$$

Let $Z$ denote the projection $U \times \mathrm{SO}_{3}(\mathbb{R}) \rightarrow \mathrm{SO}_{3}(\mathbb{R}) \subset \mathcal{M}_{3}(\mathbb{R})$. We consider on $\mathcal{F}$ the following matrix of 1 -forms:

$$
\Theta=Z^{-1} \mathrm{~d} Z-\Omega-L(Z)
$$

where $L(Z)$ is defined in Lemma 3.5, namely for $(y, Z) \in \mathcal{F}$ we have

$$
\begin{gathered}
\Theta_{(y, Z)}: \mathrm{T}_{(y, Z)} \mathcal{F} \rightarrow \mathcal{M}_{3}(\mathbb{R}), \\
\Theta_{(y, Z)}(u, \zeta)=Z^{-1} \zeta-\Omega_{y}(u)-L(Z)(u) .
\end{gathered}
$$

We claim that, for each $(y, Z) \in \mathcal{F}$, the space

$$
\mathscr{D}(y, Z)=\operatorname{ker} \Theta_{(y, Z)}
$$

has dimension 2. We first notice that the matrix $\Theta$ belongs to $\mathfrak{s o}_{3}(\mathbb{R})$ since $\Omega, L(Z)$ and $Z^{-1} \mathrm{~d} Z$ do. Moreover we have

$$
(Z \Theta)_{\beta}^{3}=\mathrm{d} Z_{\beta}^{3}-\sum_{\gamma} Z_{\gamma}^{3} \omega_{\beta}^{\gamma}-\sum_{\gamma} Z_{\gamma}^{3} L(Z)_{\beta}^{\gamma}=\mathrm{d} T^{\beta}-\sum_{\gamma} T^{\gamma} \omega_{\beta}^{\gamma}-\sum_{\gamma} T^{\gamma} L(Z)_{\beta}^{\gamma} .
$$

Using Lemmas 4.5 and 4.7 we compute that

$$
(Z \Theta)_{\beta}^{3}=0 .
$$

Thus the values of $\Theta_{(y, Z)}$ lie in the space

$$
\mathscr{H}=\left\{H \in \mathfrak{s o}_{3}(\mathbb{R}) ;(Z H)_{\beta}^{3}=0\right\},
$$

which has dimension 1 (indeed, the map $F: \mathrm{SO}_{3}(\mathbb{R}) \rightarrow \mathbb{S}^{2}, Z \mapsto\left(Z_{\beta}^{3}\right)_{\beta}$ is a submersion, and we have $H \in \mathscr{H}$ if and only if $\left.Z H \in \operatorname{ker}(\mathrm{d} F)_{Z}\right)$. Moreover, the space $\mathrm{T}_{(y, Z)} \mathcal{F}$ contains the subspace $\{(0, Z H) ; H \in \mathscr{H}\}$, and the restriction of $\Theta_{(y, Z)}$ on this subspace is the map $(0, Z H) \mapsto H$. Thus $\Theta_{(y, Z)}$ is onto $\mathscr{H}$, and consequently the linear map $\Theta_{(y, Z)}$ has rank 1. This finishes proving the claim.

We now prove that the distribution $\mathscr{D}$ is involutive. We first compute that

$$
\begin{aligned}
\mathrm{d} \Theta= & -Z^{-1} \mathrm{~d} Z \wedge Z^{-1} \mathrm{~d} Z-\mathrm{d} \Omega-\mathrm{d} L \\
= & -(\Theta+\Omega+L) \wedge(\Theta+\Omega+L)-\mathrm{d} \Omega-\mathrm{d} L \\
= & -\Theta \wedge \Theta-\Theta \wedge \Omega-\Omega \wedge \Theta-\Theta \wedge L-L \wedge \Theta \\
& \quad-\Omega \wedge L-L \wedge \Omega-\Omega \wedge \Omega-\mathrm{d} \Omega-L \wedge L-\mathrm{d} L .
\end{aligned}
$$


Using Lemmas 4.4, 4.6, 4.8, 4.9 and the relation $\sigma=\frac{\kappa}{2 \tau}$, we obtain

$$
\mathrm{d} \Theta=-\Theta \wedge \Theta-\Theta \wedge \Omega-\Omega \wedge \Theta-\Theta \wedge L-L \wedge \Theta .
$$

From this formula we deduce that if $\xi_{1}, \xi_{2} \in \mathcal{D}$, then $\mathrm{d} \Theta\left(\xi_{1}, \xi_{2}\right)=0$, and so $\Theta\left(\left[\xi_{1}, \xi_{2}\right]\right)=\xi_{1} \cdot \Theta\left(\xi_{2}\right)-\xi_{2} \cdot \Theta\left(\xi_{1}\right)-\mathrm{d} \Theta\left(\xi_{1}, \xi_{2}\right)=0$, i.e., $\left[\xi_{1}, \xi_{2}\right] \in \mathscr{D}$. Thus the distribution $\mathscr{D}$ is involutive, and so, by the theorem of Frobenius, it is integrable.

Let $\mathcal{A}$ be the integral manifold through $\left(y_{0}, A_{0}\right)$. If $\zeta \in \mathrm{T}_{A_{0}} \mathrm{SO}_{3}(\mathbb{R})$ is such that $(0, \zeta) \in \mathrm{T}_{\left(y_{0}, A_{0}\right)} \mathcal{A}=\mathscr{D}\left(y_{0}, A_{0}\right)$, then we have $0=\Theta_{\left(y_{0}, A_{0}\right)}(0, \zeta)=A_{0}^{-1} \zeta$. This proves that

$$
\mathrm{T}_{\left(y_{0}, A_{0}\right)} \mathcal{A} \cap\left(\{0\} \times \mathrm{T}_{A_{0}} \mathrm{SO}_{3}(\mathbb{R})\right)=\{0\} .
$$

Thus the manifold $\mathcal{A}$ is locally the graph of a function $A: U_{1} \rightarrow \mathrm{SO}_{3}(\mathbb{R})$ where $U_{1}$ is a neighbourhood of $y_{0}$ in $U$. By construction, this map satisfies the properties of Proposition 4.10 and is unique.

Proposition 4.11. Let $x_{0} \in \mathbb{E}$ (without loss of generality we can assume that $\left.x_{0} \in \mathbb{E}^{\prime}\right)$. There exist a neighbourhood $U_{2}$ of $y_{0}$ contained in $U_{1}$ and a unique function $f: U_{2} \rightarrow \mathbb{E}^{\prime}$ such that

$$
\begin{gathered}
\mathrm{d} f=(B \circ f) A \omega, \\
f\left(y_{0}\right)=x_{0},
\end{gathered}
$$

where $\omega$ is the column $\left(\omega^{1}, \omega^{2}, 0\right)$ and, for $x \in \mathbb{E}^{\prime}, B(x) \in \mathcal{M}_{3}(\mathbb{R})$ is the matrix of the coordinates of the frame $\left(E_{\alpha}(x)\right)$ in the frame $\left(\partial_{x^{\alpha}}\right)$.

Proof. We consider on $U_{1} \times \mathbb{E}^{\prime}$ the following matrix of 1-forms:

$$
\Lambda=B^{-1} \mathrm{~d} x-A \omega,
$$

namely, for $q \in U_{1}$ and $x \in \mathbb{E}^{\prime}$ we have

$$
\begin{gathered}
\Lambda_{(q, x)}: \mathrm{T}_{q} U_{1} \oplus \mathrm{T}_{x} \mathbb{E} \rightarrow \mathcal{M}_{3,1}(\mathbb{R}), \\
\Lambda_{(q, x)}(u, v)=B(x)^{-1} v-A(q) \omega_{q}(u) .
\end{gathered}
$$

We first notice that for all $(q, x) \in U_{1} \times \mathbb{E}^{\prime}$ the linear map $\Lambda_{(q, x)}$ is onto $\mathcal{M}_{3,1}(\mathbb{R})$. Consequently the space

$$
\mathcal{E}(q, x)=\operatorname{ker} \Lambda_{(q, x)}
$$

has dimension 2 . We will prove that this distribution $\&$ is integrable.

We have

$$
\mathrm{d} \Lambda=-B^{-1} \mathrm{~d} B B^{-1} \wedge \mathrm{d} x-\mathrm{d} A \wedge \omega-A \wedge \mathrm{d} \omega .
$$


By equations (4) and (5) we have $\mathrm{d} \omega=-\Omega \wedge \omega$; and by Proposition 4.10 we have $\mathrm{d} A=A \Omega+A L(A)$. Thus we get

$$
\mathrm{d} \Lambda=-B^{-1} \mathrm{~d} B \wedge \Lambda-B^{-1} \mathrm{~d} B \wedge A \omega-A L(A) \wedge \omega .
$$

Using Lemma 4.7 we compute that

$$
L(A) \wedge \omega=-(2 \tau-\sigma) T^{3}\left(\begin{array}{l}
T^{1} \\
T^{2} \\
T^{3}
\end{array}\right) \omega^{1} \wedge \omega^{2}-\left(\begin{array}{l}
0 \\
0 \\
\sigma
\end{array}\right) \omega^{1} \wedge \omega^{2},
$$

and thus, using the fact that $A_{\beta}^{3}=T^{\beta}$ and $A=\operatorname{com} A$, we get

$$
A L(A) \wedge \omega=\left(\begin{array}{c}
-\sigma A_{3}^{1} \\
-\sigma A_{3}^{2} \\
-2 \tau T^{3}
\end{array}\right) \omega^{1} \wedge \omega^{2} .
$$

We will use the notation $(x, y, z)$ instead of $\left(x^{1}, x^{2}, x^{3}\right)$ for the coordinates in $\mathbb{E}$ and we will use the local models described in Sections 2.2, 2.3 and 2.4. Using formulas (1), (2) and (3), we get that the matrix $B$ is

$$
B=\left(\begin{array}{ccc}
\lambda^{-1} \cos (\sigma z) & -\lambda^{-1} \sin (\sigma z) & 0 \\
\lambda^{-1} \sin (\sigma z) & \lambda^{-1} \cos (\sigma z) & 0 \\
\tau(x \sin \sigma z-y \cos \sigma z) & \tau(x \cos \sigma z+y \sin \sigma z) & 1
\end{array}\right),
$$

with

$$
\lambda=\frac{1}{1+\frac{\kappa}{4}\left(x^{2}+y^{2}\right)} .
$$

We will write

$$
A \omega=\left(\begin{array}{c}
\alpha^{1} \\
\alpha^{2} \\
\eta
\end{array}\right)
$$

with

$$
\alpha^{j}=A_{1}^{j} \omega^{1}+A_{2}^{j} \omega^{2}
$$

Then we have

$$
\Lambda=B^{-1} \mathrm{~d} X-A \omega=\left(\begin{array}{c}
\lambda(\cos (\sigma z) \mathrm{d} x+\sin (\sigma z) \mathrm{d} y)-\alpha^{1} \\
\lambda(-\sin (\sigma z) \mathrm{d} x+\cos (\sigma z) \mathrm{d} y)-\alpha^{2} \\
\tau \lambda(y \mathrm{~d} x-x \mathrm{~d} y)+\mathrm{d} z-\eta
\end{array}\right) .
$$

We also compute that

$$
B^{-1} \mathrm{~d} B=\left(\begin{array}{ccc}
\frac{\kappa}{2} \lambda(x \mathrm{~d} x+y \mathrm{~d} y) & -\sigma \mathrm{d} z & 0 \\
\sigma \mathrm{d} z & \frac{\kappa}{2} \lambda(x \mathrm{~d} x+y \mathrm{~d} y) & 0 \\
a & b & 0
\end{array}\right)
$$


with

$$
\begin{aligned}
a & =\frac{\tau \kappa}{2} \lambda(y \cos (\sigma z)-x \sin (\sigma z))(x \mathrm{~d} x+y \mathrm{~d} y)+\tau(\sin (\sigma z) \mathrm{d} x-\cos (\sigma z) \mathrm{d} y), \\
b & =-\frac{\tau \kappa}{2} \lambda(x \cos (\sigma z)+y \sin (\sigma z))(x \mathrm{~d} x+y \mathrm{~d} y)+\tau(\cos (\sigma z) \mathrm{d} x+\sin (\sigma z) \mathrm{d} y) .
\end{aligned}
$$

Thus we have

$$
\begin{aligned}
& B^{-1} \mathrm{~d} B \wedge A \omega+A L(A) \wedge \omega=\left(\begin{array}{c}
\frac{\kappa}{2} \lambda(x \mathrm{~d} x+y \mathrm{~d} y) \wedge \alpha^{1}-\sigma \mathrm{d} z \wedge \alpha^{2} \\
\sigma \mathrm{d} z \wedge \alpha^{1}+\frac{\kappa}{2} \lambda(x \mathrm{~d} x+y \mathrm{~d} y) \wedge \alpha^{2} \\
a \wedge \alpha^{1}+b \wedge \alpha^{2}
\end{array}\right) \\
&+\left(\begin{array}{c}
-\sigma A_{3}^{1} \\
-\sigma A_{3}^{2} \\
-2 \tau T^{3}
\end{array}\right) \omega^{1} \wedge \omega^{2} .
\end{aligned}
$$

Using the above expression for $\Lambda$ we get

$$
\begin{gathered}
\lambda \mathrm{d} x=\cos (\sigma z) \Lambda^{1}-\sin (\sigma z) \Lambda^{2}+\cos (\sigma z) \alpha^{1}-\sin (\sigma z) \alpha^{2}, \\
\lambda \mathrm{d} y=\sin (\sigma z) \Lambda^{1}+\cos (\sigma z) \Lambda^{2}+\sin (\sigma z) \alpha^{1}+\cos (\sigma z) \alpha^{2}, \\
\mathrm{~d} z=\Lambda^{3}+\eta-\tau \lambda(y \mathrm{~d} x-x \mathrm{~d} y) .
\end{gathered}
$$

The term in the first line of the matrix $B^{-1} \mathrm{~d} B \wedge A \omega+A L(A)$ is

$$
\begin{array}{r}
\frac{\kappa}{2}(y \cos (\sigma z)-x \sin (\sigma z)) \alpha^{2} \wedge \alpha^{1}+\sigma \tau(y \cos (\sigma z)-x \sin (\sigma z)) \alpha^{1} \wedge \alpha^{2} \\
-\sigma \eta \wedge \alpha^{2}-\sigma A_{3}^{1} \omega^{1} \wedge \omega^{2}+\chi^{1}
\end{array}
$$

where $\chi^{1}$ is a linear combination of the $\Lambda^{\alpha}$ (the coefficients being 1-forms). Since $\sigma=\frac{\kappa}{2 \tau}$, the first two terms in this expression cancel. Moreover we have $\eta \wedge \alpha^{2}=$ $\left(A_{1}^{3} A_{2}^{2}-A_{2}^{3} A_{1}^{2}\right) \omega^{1} \wedge \omega^{2}=-A_{3}^{1} \omega^{1} \wedge \omega^{2}$, hence the term in the first line of the matrix $B^{-1} \mathrm{~d} B \wedge A \omega+A L(A)$ is $\chi^{1}$. In the same way, the term in the second line of the matrix $B^{-1} \mathrm{~d} B \wedge A \omega+A L(A)$ is a linear combination of the $\Lambda^{\alpha}$ which will be denoted by $\chi^{2}$. Finally we compute that the term in the third line of the matrix $B^{-1} \mathrm{~d} B \wedge A \omega+A L(A)$ is

$$
\left(\frac{2 \tau}{\lambda}-\frac{\tau \kappa}{2}\left(x^{2}+y^{2}\right)\right) \alpha^{1} \wedge \alpha^{2}-2 \tau T^{3} \omega^{1} \wedge \omega^{2}+\chi^{3}
$$

where $\chi^{3}$ is a linear combination of the $\Lambda^{\alpha}$. Since $\lambda^{-1}=1+\frac{\kappa}{4}\left(x^{2}+y^{2}\right)$ and $\alpha^{1} \wedge \alpha^{2}=\left(A_{1}^{1} A_{2}^{2}-A_{2}^{1} A_{1}^{2}\right) \omega^{1} \wedge \omega^{2}=T^{3} \omega^{1} \wedge \omega^{2}$, this term is simply $\chi^{3}$. We conclude that

$$
B^{-1} \mathrm{~d} B \wedge A \omega+A L(A)=\chi
$$


where $\chi$ is a matrix of 2-forms which are linear combinations of the coefficients of $\Lambda$. Finally we have

$$
\mathrm{d} \Lambda=-B^{-1} \mathrm{~d} B \wedge \Lambda-\chi .
$$

From this formula we deduce that if $\xi_{1}, \xi_{2} \in \mathcal{E}$, then $\mathrm{d} \Lambda\left(\xi_{1}, \xi_{2}\right)=0$, and therefore $\left[\xi_{1}, \xi_{2}\right] \in \mathcal{E}$. Thus the distribution $\mathcal{E}$ is involutive, and so, by the theorem of Frobenius, it is integrable.

Let $\mathcal{A}$ be the integral manifold through $\left(y_{0}, x_{0}\right)$. If $v \in \mathrm{T}_{x_{0}} \mathbb{E}$ is such that $(0, v) \in$ $\mathrm{T}_{\left(y_{0}, x_{0}\right)} \mathcal{A}=\mathscr{D}\left(y_{0}, x_{0}\right)$, then we have $0=\Lambda_{\left(y_{0}, x_{0}\right)}(0, v)=B\left(x_{0}\right)^{-1} v$. This proves that

$$
\mathrm{T}_{\left(y_{0}, x_{0}\right)} \mathcal{A} \cap\left(\{0\} \times \mathrm{T}_{x_{0}} \mathbb{E}\right)=\{0\} .
$$

Thus the manifold $\mathcal{A}$ is locally the graph of a function $A: U_{2} \rightarrow \mathbb{E}^{\prime}$ where $U_{2}$ is a neighbourhood of $y_{0}$ in $U_{1}$. By construction, this map satisfies the properties of Proposition 4.10 and is unique.

We now prove the theorem.

Proof of Theorem 4.3. Let $y_{0} \in \mathcal{V}, A_{0} \in \mathcal{Z}\left(y_{0}\right)$ and $x_{0} \in \mathbb{E}^{\prime}$. We consider on $\mathcal{V}$ a local orthonormal frame $\left(e_{1}, e_{2}\right)$ in the neighbourhood of $y_{0}$ and we keep the same notations. Then by Propositions 4.10 and 4.11 there exists a unique map $A: U_{2} \rightarrow$ $\mathrm{SO}^{3}(\mathbb{R})$ such that

$$
\begin{gathered}
A^{-1} \mathrm{~d} A=\Omega+L(A), \\
A(y) \in \mathcal{Z}(y) \quad \text { for all } y \in U_{1}, \\
A\left(y_{0}\right)=A_{0},
\end{gathered}
$$

and a unique map $f: U_{2} \rightarrow \mathbb{E}^{\prime}$ such that

$$
\begin{gathered}
\mathrm{d} f=(B \circ f) A \omega, \\
f\left(y_{0}\right)=x_{0},
\end{gathered}
$$

where $U_{2}$ is a neighbourhood of $y_{0}$, which we can assume simply connected. We will check that $f$ has the properties required in the theorem on $U_{2}$.

We have $\mathrm{d} f^{\alpha}\left(e_{k}\right)=(B(f) A)_{k}^{\alpha}$, so in the frame $\left(\partial_{x^{\alpha}}\right)$ the vector $\mathrm{d} f\left(e_{k}\right)$ is given by the column $k$ of the matrix $B A$, which is invertible. Hence $\mathrm{d} f$ has rank 2 , and thus $f$ is an immersion. Moreover, in the frame $\left(E_{\alpha}\right)$ the vector $\mathrm{d} f\left(e_{k}\right)$ is given by the column $k$ of the matrix $A$, which is orthogonal, and thus we have $\left\langle\mathrm{d} f\left(e_{p}\right), \mathrm{d} f\left(e_{q}\right)\right\rangle=\delta_{q}^{p}$, which means that $f$ is an isometry.

The columns of $A(y)$ form a direct orthonormal frame of $\mathbb{E}$. The first and second columns form a direct orthonormal frame of $\mathrm{T}_{f(y)} f(\mathcal{V})$ Thus the third column gives, in the frame $\left(E_{\alpha}\right)$, the unit normal $N(f(y))$ to $f(\mathcal{V})$ in $\mathbb{E}$ at the point $f(y)$. 
We set $X_{j}=\mathrm{d} f\left(e_{j}\right)$. Then we have

$$
\begin{aligned}
\mathrm{d} A_{j}^{\alpha}\left(e_{k}\right) & =\left\langle\bar{\nabla}_{X_{k}} X_{j}, E_{\alpha}\right\rangle+\left\langle X_{j}, \bar{\nabla}_{X_{k}} E_{\alpha}\right\rangle \\
& =\left\langle\bar{\nabla}_{X_{k}} X_{j}, E_{\alpha}\right\rangle+\sum_{\gamma} \sum_{\delta} A_{k}^{\gamma} A_{j}^{\delta} \bar{\Gamma}_{\gamma \alpha}^{\delta} \\
& =\left\langle\bar{\nabla}_{X_{k}} X_{j}, E_{\alpha}\right\rangle+(A L(A))_{j}^{\alpha}\left(e_{k}\right),
\end{aligned}
$$

so

$$
\begin{aligned}
\left\langle\bar{\nabla}_{X_{k}} X_{j}, N\right\rangle & =\sum_{\alpha}\left\langle\bar{\nabla}_{X_{k}} X_{j}, E_{\alpha}\right\rangle A_{3}^{\alpha}=\sum_{\alpha} A_{3}^{\alpha}(\mathrm{d} A-A L(A))_{j}^{\alpha}\left(e_{k}\right) \\
& =\sum_{\alpha} A_{3}^{\alpha}(A \Omega)_{j}^{\alpha}\left(e_{k}\right)=\sum_{\alpha} \sum_{\gamma} A_{\gamma}^{\alpha} A_{3}^{\alpha} \omega_{j}^{\gamma}\left(e_{k}\right) \\
& =\omega_{j}^{3}\left(e_{k}\right)=\left\langle\mathrm{S} e_{k}, e_{j}\right\rangle .
\end{aligned}
$$

This means that the shape operator of $f(\mathcal{V})$ in $\mathbb{E}$ is $\mathrm{d} f \circ \mathrm{S} \circ \mathrm{d} f^{-1}$.

Finally, the coefficients of the vertical vector $\xi=E_{3}$ in the orthonormal frame $\left(X_{1}, X_{2}, N\right)$ are given by the last line of $A$. Since $A(y) \in \mathcal{Z}(y)$ for all $y \in U_{2}$ we get

$$
\xi=\sum_{j} T^{j} X_{j}+T^{3} N=\mathrm{d} f(T)+v N .
$$

We now prove that the local immersion is unique up to a global isometry of $\mathbb{E}$ preserving $\xi$ (and also, consequently, the orientation of the base of the fibration). Let $\tilde{f}: U_{3} \rightarrow \mathbb{E}$ be another immersion satisfying the conclusion of the theorem, where $U_{3}$ is a simply connected neighbourhood of $y_{0}$ included in $U_{2}$, let $\left(\tilde{X}_{\beta}\right)$ be the associated frame (i.e., $\tilde{X}_{j}=\mathrm{d} \tilde{f}\left(e_{j}\right)$ and $\tilde{X}_{3}$ is the normal of $\left.\tilde{f}(\mathcal{V})\right)$ and let $\tilde{A}$ the matrix of the coordinates of the frame $\left(\tilde{X}_{\beta}\right)$ in the frame $\left(E_{\alpha}\right)$. Up to an isometry of $\mathbb{E}$ (which is necessarily direct), we can assume that $f\left(y_{0}\right)=\tilde{f}\left(y_{0}\right)$ and that the frames $\left(X_{\beta}\left(y_{0}\right)\right)$ and $\left(\tilde{X}_{\beta}\left(y_{0}\right)\right)$ coincide, i.e., $A\left(y_{0}\right)=\tilde{A}\left(y_{0}\right)$. We notice that this isometry necessarily fixes $\xi$ since the $T^{\alpha}$ are the same for $x$ and $\tilde{x}$. The matrices $A$ and $\tilde{A}$ satisfy $A^{-1} \mathrm{~d} A=\Omega+L(\tilde{\tilde{A}})$ and $\tilde{A}^{-1} \mathrm{~d} \tilde{A}=\Omega+L(\tilde{A})$ (see Section 3.3), $A(y), \tilde{A}(y) \in \mathbb{Z}(y)$ and $A\left(y_{0}\right)=\tilde{A}\left(y_{0}\right)$, thus by the uniqueness of the solution of the equation in Proposition 4.10 we get $A(y)=\tilde{A}(y)$. We conclude similarly that $f=\tilde{f}$ on $U_{3}$.

The proof that this local immersion $f$ can be extended to the whole $\mathcal{V}$ (since $\mathcal{V}$ is simply connected) is exactly the same as the proof of the corresponding statement in Theorem 3.3 in [Dan04] (it is a standard argument).

Remark 4.12. If $\left(\mathrm{d} s^{2}, \mathrm{~S}, T, v\right)$ satisfies the compatibility equations and correspond to an immersion $f: \Sigma \rightarrow \mathbb{E}$, then $\left(\mathrm{d} s^{2}, \mathrm{~S},-T,-v\right)$ also satisfies the compatibility equations and corresponds to the immersion $\sigma \circ f$ where $\sigma$ is an isometry of $\mathbb{E}$ reversing the orientations of both the fibers and the base of the fibration. 


\section{Constant mean curvature surfaces in 3-dimensional homogeneous manifolds}

In this section we will give applications of Theorem 4.3 to constant mean curvature surfaces (CMC) in 3-dimensional homogeneous manifolds with 4-dimensional isometry group. Abresch and Rosenberg proved that there exists a holomorphic quadratic differential for CMC surfaces in $\mathbb{S}^{2} \times \mathbb{R}$ and $\mathbb{H}^{2} \times \mathbb{R}$, generalizing the Hopf differential for CMC surfaces in 3-dimensional space forms ([AR04]). Since the Hopf differential is a very useful tool for CMC surfaces, this motivated many works on CMC surfaces in $\mathbb{S}^{2} \times \mathbb{R}$ and $\mathbb{H}^{2} \times \mathbb{R}$. Recently, Abresch and Rosenberg announced the existence of a holomorphic quadratic differential for CMC surfaces in all 3-dimensional homogeneous manifolds with 4-dimensional isometry group ([AR05]). This indicates that the theory of CMC surfaces in these manifolds may be particularly interesting.

We will consider constant mean curvature immersions of oriented surfaces. Consequently the mean curvature will be defined with a sign: it will be positive if the mean curvature vector induces the same orientation as the initial orientation, and it will be negative if the mean curvature vector induces the opposite orientation.

We will denote by I and $\mathrm{J}$ the identity and the rotation of angle $\frac{\pi}{2}$ on the tangent bundle of a surface.

5.1. A generalized Lawson correspondence. It is well known that there exists an isometric correspondence between certain simply connected CMC surfaces in space-forms ([Law70]): more precisely, every simply connected CMC $H_{1}$ surface in $\mathbb{M}^{3}\left(K_{1}\right)$ is isometric to a simply connected CMC $H_{2}$ surface in $\mathbb{M}^{3}\left(K_{2}\right)$ with $K_{1}-K_{2}=H_{2}^{2}-H_{1}^{2}$, and the shape operators of these two surfaces differ by $\left(H_{2}-H_{1}\right) \mathrm{I}$. Two such surfaces are called cousin surfaces. This correspondence is often called the Lawson correspondence. In particular, any simply connected minimal surface in $\mathbb{S}^{3}$ is isometric to a CMC 1 surface in $\mathbb{R}^{3}$, and any simply connected minimal surface in $\mathbb{R}^{3}$ is isometric to a CMC 1 surface in $\mathbb{H}^{3}$.

The Lawson correspondence is a consequence of the Gauss and Codazzi equations in the space-forms.

In this section we will use the compatibility equations for homogeneous 3-manifolds with 4-dimensional isometry group and Theorem 4.3 to prove the existence of an isometric correspondence between certain simply connected CMC surfaces in these 3-manifolds. Hence this will be a generalisation of the Lawson correspondence.

The technique will be to start with some data $\left(\mathrm{d} s^{2}, \mathrm{~S}, T, v\right)$ on a surface satisfying the compatibility equations for some homogeneous 3-manifold and to modify them in order to get data satisfying the compatibility equations for another homogeneous 3-manifold. An important fact is that the space of symmetric traceless operators is globally invariant by rotation. The easiest change is to keep $\mathrm{d} s^{2}$ and $v$, and to rotate $T$ and the traceless part of $\mathrm{S}$ by some fixed angles; the Codazzi equation then implies that we need to take the same angle for $T$ and the traceless part of S. 
Proposition 5.1. Let $\mathbb{E}_{1}$ and $\mathbb{E}_{2}$ be two 3-dimensional homogeneous manifolds with 4-dimensional isometry groups, of base curvatures $\kappa_{1}$ and $\kappa_{2}$ and bundle curvatures $\tau_{1}$ and $\tau_{2}$ respectively. Assume that

$$
\kappa_{1}-4 \tau_{1}^{2}=\kappa_{2}-4 \tau_{2}^{2} .
$$

Let $H_{1}$ and $H_{2}$ be two real numbers such that

$$
\tau_{1}^{2}+H_{1}^{2}=\tau_{2}^{2}+H_{2}^{2} .
$$

Let $\mathcal{V}$ be a surface with a quadruple $\left(\mathrm{d} s^{2}, \mathrm{~S}_{1}, T_{1}, v\right)$ satisfying the compatibility equations for $\mathbb{E}_{1}$ and such that

$$
\operatorname{tr} \mathrm{S}_{1}=2 H_{1} \text {. }
$$

Let

$$
\begin{gathered}
\theta \in \mathbb{R}, \\
T_{2}=e^{\theta \mathrm{J}} T_{1}, \\
\mathrm{~S}_{2}=e^{\theta \mathrm{J}}\left(\mathrm{S}_{1}-H_{1} \mathrm{I}\right)+H_{2} \mathrm{I} .
\end{gathered}
$$

In particular $\mathrm{S}_{2}$ is symmetric and satisfies

$$
\operatorname{tr} \mathrm{S}_{2}=2 \mathrm{H}_{2} \text {. }
$$

If the real number $\theta$ satisfies

$$
\tau_{2}+i H_{2}=e^{i \theta}\left(\tau_{1}+i H_{1}\right),
$$

then the quadruple $\left(\mathrm{d} s^{2}, \mathrm{~S}_{2}, T_{2}, v\right)$ satisfies the compatibility equations for $\mathbb{E}_{2}$.

Conversely, if the function $v$ is not identically zero and if the quadruple $\left(\mathrm{d} s^{2}, \mathrm{~S}_{2}, T_{2}, v\right)$ satisfies the compatibility equations for $\mathbb{E}_{2}$, then (12) holds.

Proof. The fact that $\mathrm{S}_{2}$ is symmetric comes from the fact that the space of symmetric traceless operators is invariant by a rotation.

We have

$$
\operatorname{det}\left(\mathrm{S}_{k}-H_{k} \mathrm{I}\right)=\operatorname{det} \mathrm{S}_{k}-H_{k}^{2}
$$

for $k=1,2$, and so

$$
\operatorname{det} \mathrm{S}_{1}=\operatorname{det} \mathrm{S}_{2}+H_{1}^{2}-H_{2}^{2} \text {. }
$$

Let $K$ be the Gauss curvature of the metric $\mathrm{d} s^{2}$. By the Gauss equation (8) we have

$$
\begin{aligned}
K & =\operatorname{det} S_{1}+\tau_{1}^{2}+\left(\kappa_{1}-4 \tau_{1}^{2}\right) v^{2} \\
& =\operatorname{det} S_{2}+H_{1}^{2}-H_{2}^{2}+\tau_{1}^{2}+\left(\kappa_{1}-4 \tau_{1}^{2}\right) v^{2} \\
& =\operatorname{det} S_{2}+\tau_{2}^{2}+\left(\kappa_{2}-4 \tau_{2}^{2}\right) v^{2}
\end{aligned}
$$


since $\kappa_{1}-4 \tau_{1}^{2}=\kappa_{2}-4 \tau_{2}^{2}$ and $\tau_{1}^{2}+H_{1}^{2}=\tau_{2}^{2}+H_{2}^{2}$. Thus the quadruple $\left(\mathrm{d} s^{2}, \mathrm{~S}_{2}, T_{2}, v\right)$ satisfies the Gauss equation for $\mathbb{E}_{2}$.

Since $\mathrm{J}$ commutes with $\nabla_{X}$ for all vector fields $X$, we have

$$
\nabla_{X} \mathrm{~S}_{2} Y-\nabla_{Y} \mathrm{~S}_{2} X-\mathrm{S}_{2}[X, Y]=e^{\theta \mathrm{J}}\left(\nabla_{X} \mathrm{~S}_{1} Y-\nabla_{Y} \mathrm{~S}_{1} X-\mathrm{S}_{1}[X, Y]\right) .
$$

On the other hand, a computation done in the proof of Proposition 4.1 in [Dan04] shows that

$$
\left\langle Y, T_{2}\right\rangle X-\left\langle X, T_{2}\right\rangle Y=e^{\theta \mathrm{J}}\left(\left\langle Y, T_{1}\right\rangle X-\left\langle X, T_{1}\right\rangle Y\right) .
$$

Hence the Codazzi equation for $\mathbb{E}_{2}$ is satisfied by $\left(\mathrm{d} s^{2}, \mathrm{~S}_{2}, T_{2}, v\right)$.

To prove that the quadruple $\left(\mathrm{d} s^{2}, \mathrm{~S}_{2}, T_{2}, v\right)$ satisfies the compatibility equations (10) and (11) for $\mathbb{E}_{2}$, it suffices to prove that

$$
\mathrm{S}_{2}-\tau_{2} \mathbf{J}=e^{\theta \mathrm{J}}\left(\mathrm{S}_{1}-\tau_{1} \mathbf{J}\right)
$$

Using the expression of $S_{2}$, equation (13) is equivalent to

$$
H_{2} \mathrm{I}-\tau_{2} \mathrm{~J}=e^{\theta \mathrm{J}}\left(H_{1} \mathrm{I}-\tau_{1} \mathrm{~J}\right) .
$$

We notice that this is a purely algebraic condition: the shape operators are not involved anymore. We consider a local direct orthonormal frame and we will identify the operators with their matrix in this frame. Then we have

$$
\mathrm{J}=\left(\begin{array}{cc}
0 & -1 \\
1 & 0
\end{array}\right)
$$

Then equation (14) is equivalent to

$$
\left\{\begin{array}{l}
H_{2}=H_{1} \cos \theta+\tau_{1} \sin \theta, \\
\tau_{2}=\tau_{1} \cos \theta-H_{1} \sin \theta,
\end{array}\right.
$$

i.e., it is equivalent to equation (12). This proves the first assertion of the theorem.

Conversely, if $\left(\mathrm{d} s^{2}, \mathrm{~S}_{2}, T_{2}, v\right)$ satisfies the compatibility equations for $\mathbb{E}_{2}$, then the compatibility equations (10) for $\left(\mathrm{d} s^{2}, \mathrm{~S}_{1}, T_{1}, v\right)$ and $\left(\mathrm{d} s^{2}, \mathrm{~S}_{2}, T_{2}, v\right)$ imply that (13) holds at every point where $v \neq 0$. If there exists a point where $v \neq 0$, this implies that (12) holds.

Theorem 5.2. Let $\mathbb{E}_{1}$ and $\mathbb{E}_{2}$ be two 3-dimensional homogeneous manifolds with 4-dimensional isometry groups, of base curvatures $\kappa_{1}$ and $\kappa_{2}$ and bundle curvatures $\tau_{1}$ and $\tau_{2}$ respectively, and such that

$$
\kappa_{1}-4 \tau_{1}^{2}=\kappa_{2}-4 \tau_{2}^{2} .
$$

Let $\xi_{1}$ and $\xi_{2}$ be the vertical vector fields of $\mathbb{E}_{1}$ and $\mathbb{E}_{2}$ respectively. 
Let $\Sigma$ be a simply connected Riemann surface and let $x_{1}: \Sigma \rightarrow \mathbb{E}_{1}$ be a conformal constant mean curvature $H_{1}$ immersion with $H_{1}^{2} \geqslant \tau_{2}^{2}-\tau_{1}^{2}$. Let $N_{1}$ be the induced normal (compatible with the orientation of $\Sigma$ ). Let $\mathrm{S}_{1}$ be the symmetric operator on $\Sigma$ induced by the shape operator of $x_{1}(\Sigma)$ associated to the normal $N_{1}$. Let $T_{1}$ be the vector field on $\Sigma$ such that $\mathrm{d} x_{1}\left(T_{1}\right)$ is the projection of $\xi_{1}$ onto $\mathrm{T}\left(x_{1}(\Sigma)\right)$. Let $v=\left\langle N_{1}, \xi_{1}\right\rangle$.

Let $H_{2} \in \mathbb{R}$ such that

$$
\tau_{1}^{2}+H_{1}^{2}=\tau_{2}^{2}+H_{2}^{2}
$$

Let $\theta \in \mathbb{R}$ such that

$$
\tau_{2}+i H_{2}=e^{i \theta}\left(\tau_{1}+i H_{1}\right) .
$$

Then there exists a conformal immersion $x_{2}: \Sigma \rightarrow \mathbb{E}_{2}$ such that:

(1) the metrics induced on $\Sigma$ by $x_{1}$ and $x_{2}$ are the same,

(2) the symmetric operator on $\Sigma$ induced by the shape operator of $x_{2}(\Sigma)$ is $e^{\theta \mathrm{J}}\left(\mathrm{S}_{1}-H_{1} \mathrm{I}\right)+H_{2} \mathrm{I}$,

(3) $\xi_{2}=\mathrm{d} x_{2}\left(e^{\theta \mathrm{J}} T_{1}\right)+v N_{2}$ where $N_{2}$ is the unit normal to $x_{2}$.

Moreover, this immersion $x_{2}$ is unique up to isometries of $\mathbb{E}_{2}$ preserving the orientations of both the fibers and the base of the fibration, and it has constant mean curvature $\mathrm{H}_{2}$.

The immersions $x_{1}$ and $x_{2}$ are called sister immersions. The number $\theta$ is called the phase of $\left(x_{1}, x_{2}\right)$.

This means that there exists an isometric correspondence between CMC $H_{1}$ simply connected surfaces in $\mathbb{E}_{1}$ and CMC $H_{2}$ simply connected surfaces in $\mathbb{E}_{2}$.

Proof. Let $\mathrm{d} s^{2}$ be the metric on $\Sigma$ induced by $x_{1}$. Then $\left(\mathrm{d} s^{2}, \mathrm{~S}_{1}, T_{1}, v\right)$ satisfies the compatibility equations for $\mathbb{E}_{1}$. Thus, by Proposition 5.1, the quadruple $\left(\mathrm{d} s^{2}, \mathrm{~S}_{2}, e^{\theta \mathrm{J}} T_{1}, v\right)$ with $\mathrm{S}_{2}=e^{\theta \mathrm{J}}\left(\mathrm{S}_{1}-H_{1} \mathrm{I}\right)+H_{2} \mathrm{I}$ also does. Thus by Theorem 4.3 there exists an immersion $x_{2}$ satisfying properties 1,2 , and 3 , and this immersion is unique up to isometries of $\mathbb{E}_{2}$ preserving the orientations of both the fibers and the base of the fibration. Moreover, we have $\operatorname{tr} \mathrm{S}_{2}=2 \mathrm{H}_{2}$, i.e., the immersion $x_{2}$ has mean curvature $\mathrm{H}_{2}$.

Figure 1 helps visualizing which classes of CMC surfaces are related by the sister surface correspondence. We start from a CMC surface in some homogeneous 3-manifold. Then we can go horizontally on the graph. We can go to the left until reaching a manifold with $\tau=0$; in this case the absolute mean curvature $|H|$ increases. We can go to the right until reaching $H=0$; in this case $|H|$ decreases.

A particularly interesting case is when $\mathbb{E}_{1}$ is the Heisenberg group $\mathrm{Nil}_{3}$ with its standard metric $\left(\kappa_{1}=0, \tau_{1}=\frac{1}{2}\right)$ and $\mathbb{E}_{2}=\mathbb{H}^{2} \times \mathbb{R}\left(\kappa_{2}=-1, \tau_{2}=0\right)$. Then CMC $H_{1}$ surfaces in $\mathrm{Nil}_{3}$ correspond isometrically to CMC $H_{2}$ surfaces in $\mathbb{H}^{2} \times \mathbb{R}$ with $H_{2}^{2}=H_{1}^{2}+\frac{1}{4}$. In particular we have the following corollary. 


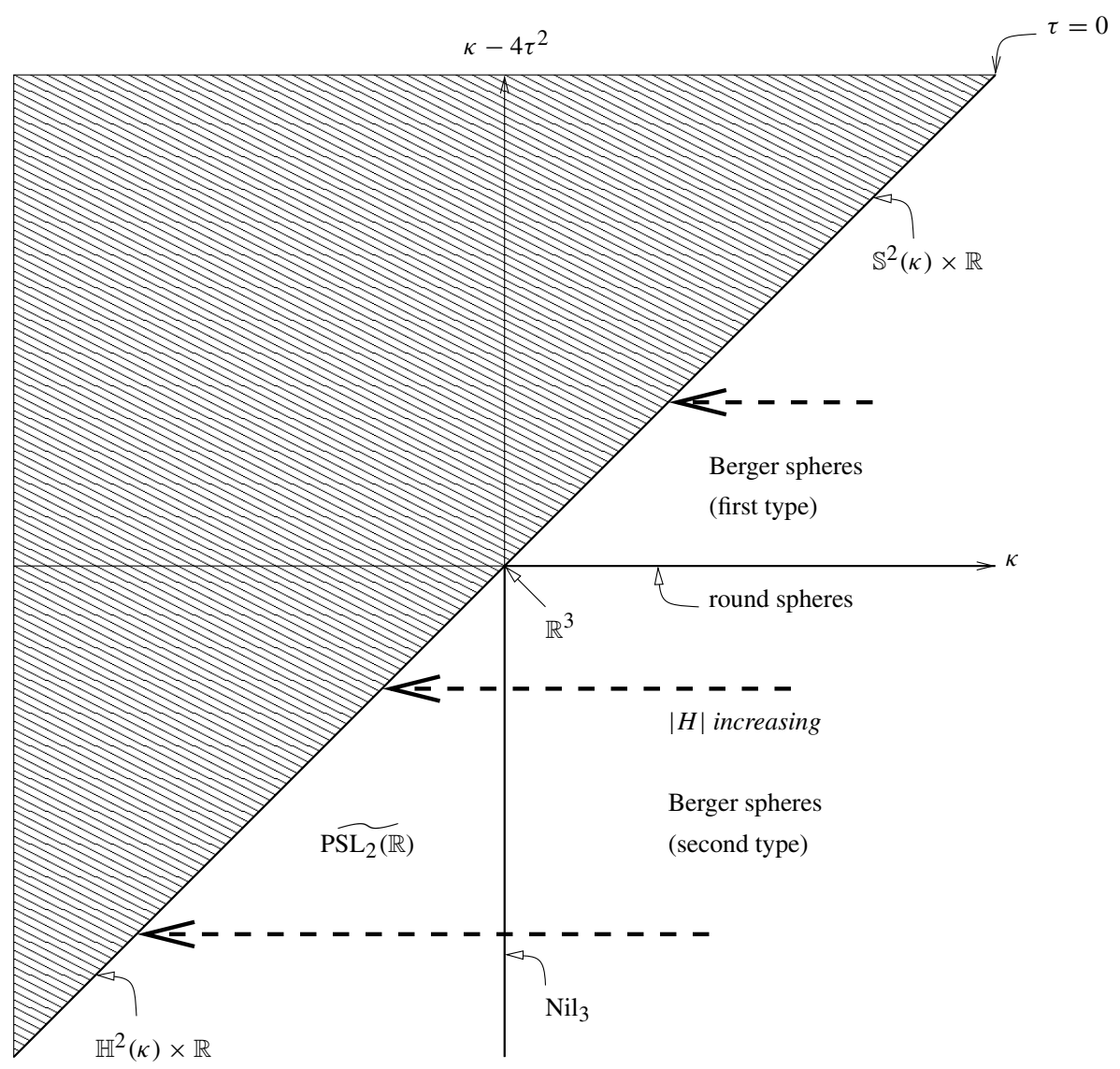

Figure 1. The correspondence of the sister surfaces.

Corollary 5.3. There exists an isometric correspondence with phase $\theta=\frac{\pi}{2}$ between simply connected minimal surfaces in the Heisenberg group $\mathrm{Nil}_{3}$ and simply connected CMC $\frac{1}{2}$ surfaces in $\mathbb{H}^{2} \times \mathbb{R}$.

The fact that $\theta=\frac{\pi}{2}$ suggests that this correspondence looks like the conjugate cousin correspondence between minimal surfaces in $\mathbb{R}^{3}$ and CMC 1 surfaces in $\mathbb{H}^{3}$ ([Bry87], [UY93]). This correspondence has nice geometric properties, and is useful to construct $\mathrm{CMC} 1$ surfaces in $\mathbb{H}^{3}$ with some prescribed geometric properties starting from a solution of a Plateau problem in $\mathbb{R}^{3}$ (see for example [Kar05], [Dan06]). In the same way, the conjugate cousin correspondence between minimal surfaces in $\mathbb{S}^{3}$ and CMC 1 surfaces in $\mathbb{R}^{3}$ was used to construct CMC 1 triunduloids in $\mathbb{R}^{3}$ ([GBKS03]). 
In particular, if a minimal surface $\Sigma_{1}$ in $\mathrm{Nil}_{3}$ contains an ambient geodesic $\gamma$, then the normal curvature of $\gamma$ vanishes, and so

$$
0=\left\langle\gamma^{\prime}, \mathrm{S}_{1} \gamma^{\prime}\right\rangle=\left\langle\gamma^{\prime},-\mathrm{JS}_{2} \gamma^{\prime}+\frac{1}{2} \mathrm{~J} \gamma^{\prime}\right\rangle=-\left\langle\gamma^{\prime}, \mathrm{JS}_{2} \gamma^{\prime}\right\rangle
$$

This means that $\mathrm{S} \gamma^{\prime}$ is colinear to $\gamma^{\prime}$, i.e., $\gamma$ is a geodesic line of curvature in the sister CMC $\frac{1}{2}$ surface in $\mathbb{H}^{2} \times \mathbb{R}$.

We describe three examples of sister CMC $\frac{1}{2}$ surfaces in $\mathbb{H}^{2} \times \mathbb{R}$ of minimal surfaces in $\mathrm{Nil}_{3}$. We will use the exponential coordinates given in Section 2.3 (with $\tau=\frac{1}{2}$ ). We will denote between parentheses ( ) the coordinates of a vector in the coordinate frame $\left(\partial_{x}, \partial_{y}, \partial_{z}\right)$, and between brackets [ ] the coordinates of a vector in the canonical frame $\left(E_{1}, E_{2}, E_{3}\right)$; with these notations one has

$$
\left(\begin{array}{l}
a \\
b \\
c
\end{array}\right)=\left[\begin{array}{c}
a \\
b \\
\frac{1}{2}(y a-x b)+c
\end{array}\right] .
$$

Example 5.4 (vertical plane). A vertical plane $\mathcal{P}$ in $\mathrm{Nil}_{3}$ is a flat minimal surface (but not totally geodesic). A conformal parametrisation is

$$
\varphi:(u, v) \mapsto\left(\begin{array}{l}
v \\
0 \\
u
\end{array}\right) .
$$

We have

$$
\varphi_{u}=E_{3}, \quad \varphi_{v}=E_{1}, \quad N=E_{2},
$$

and so

$$
\begin{gathered}
v=0, \\
\left\langle T, \partial_{u}\right\rangle=\left\langle\xi, \varphi_{u}\right\rangle=1, \\
\left\langle T, \partial_{v}\right\rangle=\left\langle\xi, \varphi_{v}\right\rangle=0,
\end{gathered}
$$

i.e.,

$$
T=\partial_{u}
$$

We also have

$$
\bar{\nabla}_{\varphi_{u}} N=\frac{1}{2} E_{1}=\frac{1}{2} \varphi_{u}, \quad \bar{\nabla}_{\varphi_{v}} N=\frac{1}{2} E_{3}=\frac{1}{2} \varphi_{v},
$$

so in the direct orthonormal frame $\left(\partial_{u}, \partial_{v}\right)$ we have

$$
\mathrm{S}=-\frac{1}{2}\left(\begin{array}{ll}
0 & 1 \\
1 & 0
\end{array}\right)
$$


We now show that the CMC $\frac{1}{2}$ sister in $\mathbb{H}^{2} \times \mathbb{R}$ of $\mathcal{P}$ is the product $\mathscr{H} \times \mathbb{R}$ where $\mathscr{H}$ is a horocycle in $\mathbb{H}^{2}$. We will use the upper half-plane model for $\mathbb{H}^{2}$. Then $\mathbb{H}^{2} \times \mathbb{R}=\left\{(x, y, z) \in \mathbb{R}^{3} ; y>0\right\}$ and the metric is $\mathrm{d} s^{2}=\frac{1}{y^{2}}\left(\mathrm{~d} x^{2}+\mathrm{d} y^{2}\right)+\mathrm{d} z^{2}$. We consider the direct orthonormal frame $\left(E_{1}, E_{2}, E_{3}\right)$ defined by $E_{1}=y \partial_{x}, E_{2}=y \partial_{y}$, $E_{3}=\partial_{z}$; it satisfies $\bar{\nabla}_{E_{1}} E_{1}=E_{2}, \bar{\nabla}_{E_{1}} E_{2}=-E_{1}$, and the other derivatives vanish. For $\mathscr{H}$, we can choose the curve of equation $y=1$ in $\mathbb{H}^{2}$. A conformal parametrization of $\mathscr{H} \times \mathbb{R}$ is

$$
\tilde{\varphi}:(u, v) \mapsto\left(\begin{array}{c}
-u \\
1 \\
v
\end{array}\right) .
$$

We have

$$
\tilde{\varphi}_{u}=-E_{1}, \quad \tilde{\varphi}_{v}=E_{3}, \quad N=E_{2},
$$

and so

$$
\tilde{v}=0, \quad \tilde{T}=\partial_{v}
$$

We also have

$$
\bar{\nabla}_{\tilde{\varphi}_{u}} N=E_{1}=-\tilde{\varphi}_{u}, \quad \bar{\nabla}_{\tilde{\varphi}_{v}} N=0,
$$

so in the direct orthonormal frame $\left(\partial_{u}, \partial_{v}\right)$ we have

$$
\tilde{\mathrm{S}}=\left(\begin{array}{ll}
1 & 0 \\
0 & 0
\end{array}\right)
$$

Hence, $\tilde{\varphi}$ induces on $\mathbb{R}^{2}$ the same metric as $\varphi$, and we have $\tilde{v}=v, \tilde{T}=\mathrm{J} T$ and $\tilde{\mathrm{S}}=\mathrm{JS}+\frac{1}{2} \mathrm{I}$, so $\tilde{\varphi}$ is the sister immersion of $\varphi$. The vertical lines in $\mathcal{P}$ are mapped to horizontal horocycles in $\mathscr{H} \times \mathbb{R}$, and horizontal lines in $\mathcal{P}$ are mapped to vertical lines in $\mathscr{H} \times \mathbb{R}$.

Example 5.5 (surface of equation $z=0$ ). The surface $\mathcal{A}$ of equation $z=0$ in the exponential coordinates is a minimal surface in $\mathrm{Nil}_{3}$ which is invariant by rotation about the $z$-axis (but it is not invariant by any translation; see [FMP99]). We consider the following parametrisation:

$$
\varphi:(u, v) \mapsto\left(\begin{array}{c}
u \cos v \\
u \sin v \\
0
\end{array}\right),
$$

for $u>0$ (the origin in $\mathcal{A}$ is excluded). We have

$$
\varphi_{u}=\left(\begin{array}{c}
\cos v \\
\sin v \\
0
\end{array}\right)=\left[\begin{array}{c}
\cos v \\
\sin v \\
0
\end{array}\right],
$$


Vol. 82 (2007)

$$
\varphi_{v}=\left(\begin{array}{c}
-u \sin v \\
u \cos v \\
0
\end{array}\right)=\left[\begin{array}{c}
-u \sin v \\
u \cos v \\
-\frac{1}{2} u^{2}
\end{array}\right]
$$

so

$$
\begin{gathered}
\left\langle\varphi_{u}, \varphi_{u}\right\rangle=1, \\
\left\langle\varphi_{v}, \varphi_{v}\right\rangle=u^{2}\left(1+\frac{u^{2}}{4}\right), \\
\left\langle\varphi_{u}, \varphi_{v}\right\rangle=0 .
\end{gathered}
$$

The unit normal vector is $N=\frac{\varphi_{u} \times \varphi_{v}}{\left\|\varphi_{u} \times \varphi_{v}\right\|}$; we compute that

$$
v=\frac{1}{\sqrt{1+\frac{u^{2}}{4}}} .
$$

A direct orthonormal frame $\left(e_{1}, e_{2}\right)$ is given by

$$
e_{1}=\partial_{u}, \quad e_{2}=\frac{1}{u \sqrt{1+\frac{u^{2}}{4}}} \partial_{v} .
$$

We compute that

$$
T=-\frac{u}{2 \sqrt{1+\frac{u^{2}}{4}}} \partial_{v} .
$$

We now show that the CMC $\frac{1}{2}$ sister in $\mathbb{H}^{2} \times \mathbb{R}$ of $\mathcal{A}$ is the CMC $\frac{1}{2}$ graph $\mathscr{B}$ of Theorem D in [NR04]. This surface $\mathcal{B}$ is a graph over the entire $\mathbb{H}^{2}$ and it is invariant by rotation about a vertical axis. If we take for $\mathbb{H}^{2}$ the Poincaré unit disk model, then $\mathcal{B}$ is the graph of the function $(x, y) \mapsto \frac{2}{\sqrt{1-x^{2}-y^{2}}}$. We will use the Lorentzian
for $\mathbb{H}^{2} \times \mathbb{R}$, i.e.,

$$
\mathbb{H}^{2} \times \mathbb{R}=\left\{\left(x^{0}, x^{1}, x^{2}, x^{3}\right) \in \mathbb{L}^{3} \times \mathbb{R} ;-\left(x^{0}\right)^{2}+\left(x^{1}\right)^{2}+\left(x^{2}\right)^{2}=-1, x_{0}>0\right\}
$$

with the restriction of the quadratic form $-\left(\mathrm{d} x^{0}\right)^{2}+\left(\mathrm{d} x^{1}\right)^{2}+\left(\mathrm{d} x^{2}\right)^{2}+\left(\mathrm{d} x^{3}\right)^{2}$. In this model, we consider the map

$$
\tilde{\varphi}:(u, v) \mapsto\left(\begin{array}{c}
1+\frac{u^{2}}{2} \\
u \sqrt{1+\frac{u^{2}}{4}} \cos v \\
u \sqrt{1+\frac{u^{2}}{4}} \sin v \\
2 \sqrt{1+\frac{u^{2}}{4}}
\end{array}\right),
$$


for $u>0$. We can check that it is a parametrization of $\mathscr{B}$ minus the origin (using that the correspondence between the Poincare model and the Lorentzian model is given by $\left.x+i y=\frac{x^{1}+i x^{2}}{1+x^{0}}, z=x^{3}\right)$. We have

$$
\tilde{\varphi}_{u}=\frac{1}{\sqrt{1+\frac{u^{2}}{4}}}\left(\begin{array}{c}
u \sqrt{1+\frac{u^{2}}{4}} \\
\left(1+\frac{u^{2}}{2}\right) \cos v \\
\left(1+\frac{u^{2}}{2}\right) \sin v \\
\frac{u}{2}
\end{array}\right), \quad \tilde{\varphi}_{v}=\left(\begin{array}{c}
0 \\
-u \sqrt{1+\frac{u^{2}}{4}} \sin v \\
u \sqrt{1+\frac{u^{2}}{4}} \cos v \\
0
\end{array}\right),
$$

so

$$
\begin{gathered}
\left\langle\tilde{\varphi}_{u}, \tilde{\varphi}_{u}\right\rangle=1, \\
\left\langle\tilde{\varphi}_{v}, \tilde{\varphi}_{v}\right\rangle=u^{2}\left(1+\frac{u^{2}}{4}\right), \\
\left\langle\tilde{\varphi}_{u}, \tilde{\varphi}_{v}\right\rangle=0,
\end{gathered}
$$

so $\tilde{\varphi}$ induces the same metric as $\varphi$. We compute that

$$
\tilde{T}=\frac{u}{2 \sqrt{1+\frac{u^{2}}{4}}} e_{1}=\mathrm{J} T .
$$

Thus we also have $\tilde{v}^{2}=v^{2}$. Moreover, $\tilde{\varphi}_{\tilde{\varphi}}$ points outwards and $\tilde{\varphi}_{v}$ points in the counter-clockwise direction, so the normal $\tilde{N}$ points up, i.e., $\tilde{v}>0$. So we get

$$
\tilde{v}=v \text {. }
$$

It remains to check that $\tilde{S}=\mathrm{JS}+\frac{1}{2} \mathrm{I}$. Since $v \neq 0$, the compatibility equations (10) for $\varphi$ and $\tilde{\varphi}$ imply that $\tilde{S}=\mathrm{J}\left(\mathrm{S}-\frac{1}{2} \mathrm{~J}\right)=\mathrm{JS}+\frac{1}{2} \mathrm{I}$. Hence $\tilde{\varphi}$ is the sister immersion of $\varphi$.

The straight lines in $\mathcal{A}$ passing through the origin are mapped to the generatrices of $\mathcal{B}$, which are lines of curvatures lying in vertical planes. Thus the symmetries of $\mathscr{B}$ with respect to these vertical planes correspond to the symmetries of $\mathscr{A}$ with respect to the straight lines passing through the origin.

Example 5.6 (surface of equation $z=\frac{x y}{2}$ ). The surface $\mathcal{A}$ of equation $z=\frac{x y}{2}$ in the exponential coordinates is a minimal surface in $\mathrm{Nil}_{3}$ which is invariant by translation along the $x$-axis (see [FMP99]; note that in this model this translation is not given by the Euclidean one). We consider the following conformal parametrisation:

$$
\varphi:(u, v) \mapsto\left(\begin{array}{c}
u \\
\sinh v \\
\frac{1}{2} u \sinh v
\end{array}\right) .
$$


We have

$$
\varphi_{u}=\left[\begin{array}{c}
1 \\
0 \\
\sinh v
\end{array}\right], \quad \varphi_{v}=\left[\begin{array}{c}
0 \\
\cosh v \\
0
\end{array}\right] .
$$

We have $\left\langle\varphi_{u}, \varphi_{u}\right\rangle=\left\langle\varphi_{v}, \varphi_{v}\right\rangle=\cosh ^{2} v$ and $\left\langle\varphi_{u}, \varphi_{v}\right\rangle=0$. The unit normal vector is

$$
N=\frac{1}{\cosh v}\left[\begin{array}{c}
-\sinh v \\
0 \\
1
\end{array}\right] \text {. }
$$

We have

$$
v=\frac{1}{\cosh v}, \quad T=\frac{\sinh v}{\cosh ^{2} v} \partial_{u} .
$$

We now show that the CMC $\frac{1}{2}$ sister in $\mathbb{H}^{2} \times \mathbb{R}$ of $\mathcal{A}$ is the CMC $\frac{1}{2}$ graph $\mathscr{B}$ given by formula (29) in [SE05]. This surface $\mathscr{B}$ is a graph over the entire $\mathbb{H}^{2}$ and it is invariant by a one-parameter family of hyperbolic transactions. If we take for $\mathbb{H}^{2}$ the upper half-plane model, then $\mathcal{B}$ is the graph of the function $(x, y) \mapsto \frac{\sqrt{x^{2}+y^{2}}}{y}$. We will use the notations of example 5.4. A conformal parametrisation of $\mathscr{B}$ is

$$
\tilde{\varphi}:(u, v) \mapsto\left(\begin{array}{c}
-e^{u} \tanh v \\
\frac{e^{u}}{\cosh v} \\
\cosh v
\end{array}\right) .
$$

We have

$$
\tilde{\varphi}_{u}=\left(\begin{array}{c}
-e^{u} \tanh v \\
\frac{e^{u}}{\cosh v} \\
0
\end{array}\right), \quad \tilde{\varphi}_{v}=\left(\begin{array}{c}
-\frac{1}{\sinh v} \\
-\tanh v \\
\sinh v
\end{array}\right),
$$

so $\left\langle\tilde{\varphi}_{u}, \tilde{\varphi}_{u}\right\rangle=\left\langle\tilde{\varphi}_{v}, \tilde{\varphi}_{v}\right\rangle=\cosh ^{2} v$ and $\left\langle\tilde{\varphi}_{u}, \tilde{\varphi}_{v}\right\rangle=0$. Hence $\tilde{\varphi}$ induces the same metric as $\varphi$. We compute that

$$
\tilde{v}=\frac{1}{\cosh v}=v
$$

and

$$
\tilde{T}=\frac{\sinh v}{\cosh ^{2} v} \partial_{v}=\mathrm{J} T .
$$

Finally, since $v \neq 0$, the compatibility equations (10) for $\varphi$ and $\tilde{\varphi}$ imply that $\tilde{S}=$ $\mathrm{J}\left(\mathrm{S}-\frac{1}{2} \mathrm{~J}\right)=\mathrm{JS}+\frac{1}{2} \mathrm{I}$. Hence $\tilde{\varphi}$ is the sister immersion of $\varphi$.

The straight lines in $\mathcal{A}$ given by $x=x_{0}$ are mapped to the generatrices of $\mathscr{B}$, which are lines of curvatures lying in vertical planes. Moreover, the $x$-axis in $\mathscr{A}$ is mapped to a horizontal geodesic of $\mathbb{H}^{2} \times \mathbb{R}$ lying in $\mathscr{B}$. Thus the symmetries of $\mathcal{B}$ with respect to vertical planes correspond to the symmetries of $\mathcal{A}$ with respect to the straight lines. 
Example 5.7 (CMC rotational spheres). The sister of the $\mathrm{CMC} H_{1}$ rotational sphere in $\mathrm{Nil}_{3}$ is the $\mathrm{CMC} \sqrt{H_{1}^{2}+\frac{1}{4}}$ rotational sphere in $\mathbb{H}^{2} \times \mathbb{R}$. Indeed, the sister of this sphere is a possibly immersed CMC sphere in $\mathbb{H}^{2} \times \mathbb{R}$, which is necessarily rotational by a theorem of Abresch and Rosenberg ([AR04]).

Remark 5.8. CMC $H$ surfaces in $\mathbb{H}^{2} \times \mathbb{R}$ have very different properties when $H \leqslant \frac{1}{2}$ and when $H>\frac{1}{2}$; for example compact embedded CMC $H$ surfaces exist only for $H>\frac{1}{2}$. The reader can refer for example to [NR04]. An explanation could be that CMC $H$ surfaces in $\mathbb{H}^{2} \times \mathbb{R}$ arise from minimal surfaces in a Berger sphere when $H>\frac{1}{2}$, in $\mathrm{Nil}_{3}$ when $H=\frac{1}{2}$, and in a space $\widehat{\mathrm{PSL}_{2}(\mathbb{R})}$ when $H<\frac{1}{2}$.

Remark 5.9. When $\kappa_{j}-4 \tau_{j}^{2}=0$, the sister relation is the composition of the classical cousin relation between the round 3 -spheres and $\mathbb{R}^{3}$ and of the conjugation by a phase $\theta$ in the associate family. The hyperbolic 3 -space does not appear in this classification since it is not a fibration over a 2-manifold of constant curvature.

Remark 5.10. When $\tau_{j}=H_{j}=0$, the sister relation gives the associate family of a minimal surface in $\mathbb{M}^{2}(\kappa) \times \mathbb{R}$ (any $\theta$ works). The associate family is a one-parameter family of minimal isometric deformations of this surface obtained by rotating the shape operator (see [Dan04]).

A natural question is that of the existence of minimal isometric deformations of a given minimal surface in a homogeneous 3-manifold $\mathbb{E}$ such that $\tau \neq 0$. The compatibility equations show that an associated family cannot be obtained in a simple way as when $\tau=0$; indeed, if the quadruple $\left(\mathrm{d} s^{2}, \mathrm{~S}, T, v\right)$ satisfies the compatibility equations for $\mathbb{E}$, then, in general, the quadruple $\left(\mathrm{d} s^{2}, e^{\theta \mathrm{J}} \mathrm{S}, e^{\theta \mathrm{J}} T, \nu\right)$ where $\theta \in \mathbb{R} \backslash$ $2 \pi \mathbb{Z}$ does not. The question of the existence of the associate family for minimal surfaces in $\mathbb{E}$ when $\tau \neq 0$ remains open.

5.2. Sister surfaces and stability. We now show that the stability operator is preserved by the sister immersion correspondence, which was not obvious a priori since the stability operator is extrinsic.

Proposition 5.11. Let $\Sigma$ be a simply connected Riemann surface and let $x: \Sigma \rightarrow \mathbb{E}$ be a CMC H immersion. Then the stability operator induced by $x$ on $\Sigma$ is

$$
\mathrm{L}=\Delta-2 K+4 H^{2}+4 \tau^{2}+\left(\kappa-4 \tau^{2}\right)\left(1+v^{2}\right)
$$

where $\Delta$ is the Laplacian of the induced metric on $\Sigma$ and $K$ the Gauss curvature of this metric. 
Proof. We recall that the stability operator is

$$
\mathrm{L}=\Delta+\|\mathrm{S}\|^{2}+\operatorname{Ric}(N)
$$

where $N$ is the unit normal to $x(\Sigma), \mathrm{S}$ its shape operator and Ric the Ricci curvature in $\mathbb{E}$ (see for example [NR04] and references therein).

Let $\left(E_{1}, E_{2}, E_{3}\right)$ be the canonical frame of $\mathbb{E}$. Then we have $\operatorname{Ric}\left(E_{1}\right)=\operatorname{Ric}\left(E_{2}\right)=$ $\kappa-2 \tau^{2}$ and $\operatorname{Ric}\left(E_{3}\right)=2 \tau^{2}$ (see Section 2.1), and so

$$
\operatorname{Ric}(N)=\left(\kappa-2 \tau^{2}\right)\left(1-v^{2}\right)+2 \tau^{2} v^{2}=\left(\kappa-4 \tau^{2}\right)\left(1-v^{2}\right)+2 \tau^{2} .
$$

On the other hand we have

$$
\|\mathrm{S}\|^{2}=4 H^{2}-2 \operatorname{det} \mathrm{S}=4 H^{2}+2 \tau^{2}+2\left(\kappa-4 \tau^{2}\right) \nu^{2}-2 K
$$

by the Gauss equation (8). Thus we get

$$
\|\mathrm{S}\|^{2}+\operatorname{Ric}(N)=4 H^{2}+4 \tau^{2}+\left(\kappa-4 \tau^{2}\right)\left(1+v^{2}\right)-2 K .
$$

Proposition 5.12. Let $\Sigma$ be a simply connected Riemann surface and let $x_{1}: \Sigma \rightarrow \mathbb{E}_{1}$ and $x_{2}: \Sigma \rightarrow \mathbb{E}_{2}$ be two sister immersions. Then $x_{1}$ and $x_{2}$ induce the same stability operator on $\Sigma$.

Proof. This follows Proposition 5.11 since $x_{1}$ and $x_{2}$ induce the same metric on $\Sigma$, $H_{1}^{2}+\tau_{1}^{2}=H_{2}^{2}+\tau_{2}^{2}$ and $\kappa_{1}-4 \tau_{1}^{2}=\kappa_{2}-4 \tau_{2}^{2}$ by hypotheses.

We recall that a CMC surface $\mathcal{V}$ (possibly with boundary) in a Riemannian 3manifold $\overline{\mathcal{V}}$ is said to be strongly stable if

$$
-\int_{\mathcal{V}} f \mathrm{~L} f \geqslant 0
$$

for all smooth functions $f$ on $\mathcal{V}$ with compact support, where $\mathrm{L}$ is the stability operator of $\mathcal{V}$. The CMC surface $\mathcal{V}$ is said to be weakly stable if (15) holds for all smooth functions $f$ on $\mathcal{V}$ with compact support and such that $\int_{\mathcal{V}} f=0$.

Corollary 5.13. Let $\Sigma_{1} \subset \mathbb{E}_{1}$ and $\Sigma_{2} \subset \mathbb{E}_{2}$ be sister surfaces (possibly with boundaries). Assume that $\Sigma_{1}$ and $\Sigma_{2}$ are simply connected. Then $\Sigma_{1}$ is strongly stable (respectively, weakly stable) if and only if $\Sigma_{2}$ is strongly stable (respectively, weakly stable).

Proof. Since $\Sigma_{1}$ and $\Sigma_{2}$ are simply connected, we can identify them globally, and the result follows from Proposition 5.12. 
5.3. Twin immersions. In this section we will study the special case of sister immersions lying in the same homogeneous 3-manifold. They necessarily have opposite mean curvatures.

Theorem 5.14. Let $\mathbb{E}$ be a homogeneous 3-manifold with a 4-dimensional isometry group, of base curvature $\kappa$ and bundle curvature $\tau$. Let $\xi$ be its vertical vector field.

Let $\Sigma$ be a simply connected Riemann surface and let $x: \Sigma \rightarrow \mathbb{E}$ be a conformal constant mean curvature $H \neq 0$ immersion. Let $N$ be the induced normal (compatible with the orientation of $\Sigma$ ). Let $\mathrm{S}$ be the symmetric operator on $\Sigma$ induced by the shape operator of $x(\Sigma)$ associated to the normal $N$. Let $T$ be the vector field on $\Sigma$ such that $\mathrm{d} x(T)$ is the projection of $\xi$ onto $\mathrm{T}(x(\Sigma))$. Let $v=\langle N, \xi\rangle$. Let

$$
\theta=-2 \arctan \frac{H}{\tau}
$$

Then there exists a unique conformal immersion $\hat{x}: \Sigma \rightarrow \mathbb{E}$ such that:

(1) the metrics induced on $\Sigma$ by $x$ and $\hat{x}$ are the same,

(2) the symmetric operator on $\Sigma$ induced by the shape operator of $\hat{x}(\Sigma)$ is $\tilde{\mathrm{S}}=$ $e^{\theta \mathrm{J}}(\mathrm{S}-H \mathrm{I})-H \mathrm{I}=e^{\theta \mathrm{J}}(\mathrm{S}-\tau \mathrm{J})+\tau \mathrm{J}$,

(3) $\xi=\mathrm{d} \hat{x}\left(e^{\theta \mathrm{J}} T\right)+v \hat{N}$ where $\hat{N}$ is the unit normal to $\hat{x}$.

Moreover, this immersion $\hat{x}$ is unique up to isometries of $\mathbb{E}$ preserving the orientations of both the fibers and the base of the fibration, and it has constant mean curvature $-H$.

It is called the twin immersion of the immersion $x$.

Proof. This is a particular case of Theorem 5.2 with $\mathbb{E}_{1}=\mathbb{E}_{2}=\mathbb{E}, \tau_{1}=\tau_{2}=\tau$, $H_{1}=-H_{2}=H$. It suffices to check that the phase $\theta$ satisfies $\tau-i H=e^{i \theta}(\tau+i H)$.

The equivalence of the two expressions of $\tilde{S}$ is a consequence of (14).

We notice that when $\tau \rightarrow 0$, then $\theta \rightarrow \pi$, i.e., $\tilde{T} \rightarrow-T$, and also $\tilde{\mathrm{S}} \rightarrow-\mathrm{S}$. This limit corresponds to the image of the initial surface by a horizontal symmetry in $\mathbb{M}^{2}(\kappa) \times \mathbb{R}$.

Moreover, we notice that the twin surface of a multigraph (over a part of the base of the fibration) is also a multigraph (since a surface is a multigraph if and only if $v$ does not vanish).

This suggests that the twin surface could be used to get an Alexandrov reflectiontype principle in homogeneous manifolds with non-vanishing bundle curvature, since there is no Alexandrov reflection principle (see [Ale62]) in these manifolds (the horizontal and vertical "symmetries" are not isometries). Such an Alexandrov reflection principle would be very useful for the theory of CMC surfaces in homogeneous manifolds, in particular for proving that any closed embedded CMC surface in the 
Heisenberg group or in $\widetilde{\mathrm{PSL}_{2}(\mathbb{R})}$ is a rotational sphere (this was proved for CMC surfaces in $\mathbb{R}^{3}, \mathbb{H}^{3}$, a 3-hemisphere, $\mathbb{H}^{2} \times \mathbb{R}$ and a 2-hemisphere cross $\mathbb{R}$ using the Alexandrov reflection principle).

We now give some examples of twin surfaces in the Heisenberg group $\mathrm{Nil}_{3}$ with its standard metric (i.e., $\kappa=0, \tau=\frac{1}{2}$ ). We will use the exponential coordinates described in Section 2.3. Figueroa, Mercuri and Pedrosa classified CMC surfaces in $\mathrm{Nil}_{3}$ invariant by a one-parameter family of translations or rotations (see [FMP99]; note that in their article the mean curvature is defined as the trace of the shape operator, whereas in this paper it is defined as the half of the trace). We will compute the twin surfaces of these examples. We will denote between parentheses ( ) the coordinates of a vector in the coordinate frame $\left(\partial_{x}, \partial_{y}, \partial_{z}\right)$, and between brackets [ ] the coordinates of a vector in the canonical frame $\left(E_{1}, E_{2}, E_{3}\right)$.

Example 5.15 (translational tubes). Let $H>0$. The map

$$
\varphi:(u, v) \mapsto\left(\begin{array}{c}
u \\
\frac{\cos v}{2 H} \\
u \frac{\cos v}{4 H}+\frac{1}{4 H} f(v)
\end{array}\right),
$$

with

$$
f(v)=\sqrt{1+\frac{\cos ^{2} v}{4 H^{2}}} \sin v+\frac{1+4 H^{2}}{2 H} \arcsin \left(\frac{\sin v}{\sqrt{1+4 H^{2}}}\right),
$$

for $(u, v) \in \mathbb{R}^{2}$, is a CMC $H$ immersion defining a surface which is invariant by horizontal translations in the $x$-direction. This surface is an annulus, and it is a bigraph over a part of the minimal surface of equation $z=\frac{x y}{2}$; moreover it is "symmetric" with respect to this minimal surface.

We have

$$
\begin{gathered}
\varphi_{u}=\left(\begin{array}{c}
1 \\
0 \\
\frac{\cos v}{4 H}
\end{array}\right)=\left[\begin{array}{c}
1 \\
0 \\
\frac{\cos v}{2 H}
\end{array}\right], \\
0 \\
\varphi_{v}=\left(\begin{array}{c}
-\frac{\sin v}{2 H} \\
-u \frac{\sin v}{4 H}+\frac{1}{4 H} f^{\prime}(v)
\end{array}\right)=\left[\begin{array}{c}
0 \\
-\frac{\sin v}{2 H} \\
\frac{1}{4 H} f^{\prime}(v)
\end{array}\right], \\
f^{\prime}(v)=2 \cos v \sqrt{1+\frac{\cos ^{2} v}{4 H^{2}}}
\end{gathered}
$$

and so

$$
\left\langle\varphi_{u}, \varphi_{u}\right\rangle=1+\frac{\cos ^{2} v}{4 H^{2}}
$$




$$
\begin{aligned}
& \left\langle\varphi_{v}, \varphi_{v}\right\rangle=\frac{1}{4 H^{2}}\left(1+\frac{\cos ^{4} v}{4 H^{2}}\right) . \\
& \left\langle\varphi_{u}, \varphi_{v}\right\rangle=\frac{\cos ^{2} v}{4 H^{2}} \sqrt{1+\frac{\cos ^{2} v}{4 H^{2}}} .
\end{aligned}
$$

The unit normal vector is given by $N=\frac{\varphi_{u} \times \varphi_{v}}{\left\|\varphi_{u} \times \varphi_{v}\right\|}$; we compute that

$$
v=-\frac{\sin v}{\sqrt{1+\frac{\cos ^{4} v}{4 H^{2}}}} \text {. }
$$

We have

$$
\begin{gathered}
\left\langle T, \partial_{u}\right\rangle=\left\langle\xi, \varphi_{u}\right\rangle=\frac{\cos v}{2 H}, \\
\left\langle T, \partial_{v}\right\rangle=\left\langle\xi, \varphi_{v}\right\rangle=\frac{\cos v}{2 H} \sqrt{1+\frac{\cos ^{2} v}{4 H^{2}}}
\end{gathered}
$$

We notice that $v\left(u_{1},-v\right)=-v\left(u_{2}, v\right)$ for all $\left(u_{1}, u_{2}, v\right)$. This indicates that the twin immersion could be an orientation-reversing reparametrization of the surface. For this reason we set

$$
\tilde{\varphi}:(u, v) \mapsto \varphi(u+h(v),-v)=\left(\begin{array}{c}
u+h(v) \\
\frac{\cos v}{2 H} \\
(u+h(v)) \frac{\cos v}{4 H}-\frac{1}{4 H} f(v)
\end{array}\right)
$$

where $h$ is a function. This is a CMC $-H$ immersion defining globally the same surface as $\varphi$. We compute that

$$
\tilde{\varphi}_{u}=\left[\begin{array}{c}
1 \\
0 \\
\frac{\cos v}{2 H}
\end{array}\right], \quad \tilde{\varphi}_{v}=\left[\begin{array}{c}
h^{\prime}(v) \\
-\frac{\sin v}{2 H} \\
h^{\prime}(v) \frac{\cos v}{2 H}-\frac{1}{4 H} f^{\prime}(v)
\end{array}\right],
$$

and so

$$
\begin{gathered}
\left\langle\tilde{\varphi}_{u}, \tilde{\varphi}_{u}\right\rangle=1+\frac{\cos ^{2} v}{4 H^{2}} \\
\left\langle\tilde{\varphi}_{v}, \tilde{\varphi}_{v}\right\rangle=\left(1+\frac{\cos ^{2} v}{4 H^{2}}\right) h^{\prime}(v)^{2}-\frac{\cos ^{2} v}{2 H^{2}} h^{\prime}(v) \sqrt{1+\frac{\cos ^{2} v}{4 H^{2}}} \\
+\frac{1}{4 H^{2}}\left(1+\frac{\cos ^{4} v}{4 H^{2}}\right) \\
\left\langle\tilde{\varphi}_{u}, \tilde{\varphi}_{v}\right\rangle=\left(1+\frac{\cos ^{2} v}{4 H^{2}}\right) h^{\prime}(v)-\frac{\cos ^{2} v}{4 H^{2}} \sqrt{1+\frac{\cos ^{2} v}{4 H^{2}}}
\end{gathered}
$$


Thus $\tilde{\varphi}$ induces on $\mathbb{R}^{2}$ the same metric as $\varphi$ if and only if

$$
h^{\prime}(v)=\frac{\cos ^{2} v}{2 H^{2} \sqrt{1+\frac{\cos ^{2} v}{4 H^{2}}}} .
$$

We now assume that this condition is satisfied; we can also assume that $h(0)=0$. The function $h$ is increasing. We have

$$
\begin{gathered}
\tilde{v}=v, \\
\left\langle\tilde{T}, \partial_{u}\right\rangle=\left\langle\xi, \tilde{\varphi}_{u}\right\rangle=\frac{\cos v}{2 H}, \\
\left\langle\tilde{T}, \partial_{v}\right\rangle=\left\langle\xi, \tilde{\varphi}_{v}\right\rangle=\frac{\cos v}{2 H \sqrt{1+\frac{\cos ^{2} v}{4 H^{2}}}}\left(\frac{\cos ^{2} v}{4 H^{2}}-1\right) .
\end{gathered}
$$

The direct orthonormal frame $\left(e_{1}, e_{2}\right)$ obtained from the frame $\left(\partial_{u}, \partial_{v}\right)$ by the Gram-Schmidt process satisfies

$$
e_{1}=\frac{\partial_{u}}{\left\|\partial_{u}\right\|}, \quad e_{2}=\frac{-\left\langle\partial_{u}, \partial_{v}\right\rangle \partial_{u}+\left\|\partial_{u}\right\|^{2} \partial_{v}}{\left\|\partial_{u}\right\| \sqrt{\left\|\partial_{u}\right\|^{2}\left\|\partial_{u}\right\|^{2}-\left\langle\partial_{u}, \partial_{v}\right\rangle^{2}}} .
$$

A computation gives

$$
\left\|\partial_{u}\right\|^{2}\left\|\partial_{u}\right\|^{2}-\left\langle\partial_{u}, \partial_{v}\right\rangle^{2}=\frac{1}{4 H^{2}}\left(1+\frac{\cos ^{2} v}{4 H^{2}}\right) .
$$

Thus we get

$$
e_{1}=\frac{1}{\sqrt{1+\frac{\cos ^{2} v}{4 H^{2}}}} \partial_{u}, \quad e_{2}=-\frac{\cos ^{2} v}{2 H \sqrt{1+\frac{\cos ^{2} v}{4 H^{2}}}} \partial_{u}+2 H \partial_{v} .
$$

So we have

$$
\begin{aligned}
& T=\frac{\cos v}{\sqrt{1+\frac{\cos ^{2} v}{4 H^{2}}}}\left(\frac{1}{2 H} e_{1}+e_{2}\right), \\
& \tilde{T}=\frac{\cos v}{\sqrt{1+\frac{\cos ^{2} v}{4 H^{2}}}}\left(\frac{1}{2 H} e_{1}-e_{2}\right) .
\end{aligned}
$$

Let $\theta=-2 \arctan (2 H)$. Then we have

$$
\cos \theta=\frac{1-4 H^{2}}{1+4 H^{2}}, \quad \sin \theta=-\frac{4 H}{1+4 H^{2}} .
$$


Since $\mathrm{J} e_{1}=e_{2}$ and $\mathrm{J} e_{2}=-e_{1}$, we get

$$
e^{\theta \mathrm{J}} T=\tilde{T}
$$

Finally, the compatibility equation (10) implies that

$$
\tilde{S}=e^{\theta \mathbf{J}}(\mathbf{S}-\tau \mathbf{J})+\tau \mathbf{J}
$$

at points where $v \neq 0$; and by continuity this identity holds everywhere. This proves that $\tilde{\varphi}$ is the twin immersion of $\varphi$.

Thus the translational tube is globally invariant by the twin relation, but it is not pointwise invariant: the correspondence is

$$
\varphi(u, v) \mapsto \varphi(u+h(v),-v) .
$$

Geometrically, this correspondence maps a point of the tube to the other point of the tube lying in the same fiber and then translates it by $h(v)$ in the $x$-direction. In particular, the closed curve $v \mapsto \varphi\left(u_{0}, v\right)$ is mapped to the curve $v \mapsto \varphi\left(u_{0}+\right.$ $h(v),-v)$, which is not closed.

Example 5.16 (rotational spheres). Let $H>0$. The map

$$
\varphi:(u, v) \mapsto\left(\begin{array}{c}
\frac{1}{H} \cos u \cos v \\
\frac{1}{H} \sin u \cos v \\
\frac{1}{2 H} f(v)
\end{array}\right),
$$

with $f$ as in example 5.15, for $(u, v) \in \mathbb{R} \times\left(-\frac{\pi}{2}, \frac{\pi}{2}\right)$, is a CMC $-H$ immersion defining a rotational sphere minus the top and bottom points (the normal of the immersion points outside whereas the mean curvature vector points inside). It is a bigraph over a part of the minimal surface of equation $z=0$; moreover it is "symmetric" with respect to this minimal surface.

We have

$$
\varphi_{u}=\frac{1}{H}\left[\begin{array}{c}
-\sin u \cos v \\
\cos u \cos v \\
-\frac{1}{2 H} \cos ^{2} v
\end{array}\right], \quad \varphi_{v}=\frac{1}{H}\left[\begin{array}{c}
-\cos u \sin v \\
-\sin u \sin v \\
\frac{1}{2} f^{\prime}(v)
\end{array}\right],
$$

and so

$$
\begin{gathered}
\left\langle\varphi_{u}, \varphi_{u}\right\rangle=\frac{\cos ^{2} v}{H^{2}}\left(1+\frac{\cos ^{2} v}{4 H^{2}}\right), \\
\left\langle\varphi_{v}, \varphi_{v}\right\rangle=\frac{1}{H^{2}}\left(1+\frac{\cos ^{2} v}{4 H^{2}}\right)
\end{gathered}
$$




$$
\left\langle\varphi_{u}, \varphi_{v}\right\rangle=-\frac{\cos ^{3} v}{2 H^{3}} \sqrt{1+\frac{\cos ^{2} v}{4 H^{2}}} .
$$

The unit normal vector is given by $N=\frac{\varphi_{u} \times \varphi_{v}}{\left\|\varphi_{u} \times \varphi_{v}\right\|}$; we compute that

$$
v=\frac{\sin v}{\sqrt{1+\frac{\cos ^{4} v}{4 H^{2}}}} .
$$

We have

$$
\begin{gathered}
\left\langle T, \partial_{u}\right\rangle=\left\langle\xi, \varphi_{u}\right\rangle=-\frac{\cos ^{2} v}{2 H^{2}} \\
\left\langle T, \partial_{v}\right\rangle=\left\langle\xi, \varphi_{v}\right\rangle=\frac{\cos v}{H} \sqrt{1+\frac{\cos ^{2} v}{4 H^{2}}} .
\end{gathered}
$$

Let

$$
\tilde{\varphi}:(u, v) \mapsto \varphi(u+g(v),-v)=\left(\begin{array}{c}
\frac{1}{H} \cos (u+g(v)) \cos v \\
\frac{1}{H} \sin (u+g(v)) \cos v \\
-\frac{1}{2 H} f(v)
\end{array}\right)
$$

where $g$ is a function. This is a CMC $H$ immersion defining globally the same surface as $\varphi$. We compute that

$$
\begin{gathered}
\tilde{\varphi}_{u}=\frac{1}{H}\left[\begin{array}{c}
-\sin (u+g(v)) \cos v \\
\cos (u+g(v)) \cos v \\
-\frac{1}{2 H} \cos ^{2} v
\end{array}\right], \\
\tilde{\varphi}_{v}=\frac{1}{H}\left[\begin{array}{c}
-\cos (u+g(v)) \sin v-g^{\prime}(v) \sin (u+g(v)) \cos v \\
-\sin (u+g(v)) \sin v+g^{\prime}(v) \cos (u+g(v)) \cos v \\
-\frac{1}{2} f^{\prime}(v)-\frac{1}{2 H} g^{\prime}(v) \cos ^{2} v
\end{array}\right],
\end{gathered}
$$

and thus $\tilde{\varphi}$ induces on $\mathbb{R} \times\left(\frac{\pi}{2}, \frac{\pi}{2}\right)$ the same metric as $\varphi$ if and only if

$$
g^{\prime}(v)=-\frac{\cos v}{H \sqrt{1+\frac{\cos ^{2} v}{4 H^{2}}}} .
$$

We now assume that this condition is satisfied; we can also assume that $g(0)=0$, which gives

$$
g(v)=-2 \arcsin \left(\frac{\sin v}{\sqrt{1+4 H^{2}}}\right) .
$$

The function $g$ is odd and $2 \pi$-periodic. We have

$$
\tilde{v}=v,
$$




$$
\begin{gathered}
\left\langle\tilde{T}, \partial_{u}\right\rangle=\left\langle\xi, \tilde{\varphi}_{u}\right\rangle=-\frac{\cos ^{2} v}{2 H^{2}} \\
\left\langle\tilde{T}, \partial_{v}\right\rangle=\left\langle\xi, \tilde{\varphi}_{v}\right\rangle=\frac{\cos v}{H \sqrt{1+\frac{\cos ^{2} v}{4 H^{2}}}}\left(\frac{\cos ^{2} v}{4 H^{2}}-1\right) .
\end{gathered}
$$

The direct orthonormal frame $\left(e_{1}, e_{2}\right)$ obtained from the frame $\left(\partial_{u}, \partial_{v}\right)$ by the Gram-Schmidt process satisfies

$$
e_{1}=\frac{H}{\cos v \sqrt{1+\frac{\cos ^{2} v}{4 H^{2}}}} \partial_{u}, \quad e_{2}=-\frac{\cos v}{2 \sqrt{1+\frac{\cos ^{2} v}{4 H^{2}}}} \partial_{u}+H \partial_{v} .
$$

So we have

$$
\begin{aligned}
& T=\frac{\cos v}{\sqrt{1+\frac{\cos ^{2} v}{4 H^{2}}}}\left(-\frac{1}{2 H} e_{1}+e_{2}\right), \\
& \tilde{T}=\frac{\cos v}{\sqrt{1+\frac{\cos ^{2} v}{4 H^{2}}}}\left(-\frac{1}{2 H} e_{1}-e_{2}\right) .
\end{aligned}
$$

Let $\theta=2 \arctan (2 H)$. We check as in example 5.15 that

$$
\begin{gathered}
e^{\theta \mathrm{J}} T=\tilde{T}, \\
\tilde{S}=e^{\theta \mathrm{J}}(\mathrm{S}-\tau \mathrm{J})+\tau \mathrm{J} .
\end{gathered}
$$

This proves that $\tilde{\varphi}$ is the twin immersion of $\varphi$.

Thus the rotational sphere is globally invariant by the twin relation, but it is not pointwise invariant: the correspondence is

$$
\varphi(u, v) \mapsto \varphi(u+g(v),-v) .
$$

Geometrically, this correspondence maps a point of the sphere to the other point of the sphere lying in the same fiber and then rotates it by the angle $g(v)$ about the $z$-axis. In particular, the circle $v \mapsto \varphi\left(u_{0}, v\right)$ lying in a vertical plane is mapped to the curve $v \mapsto \varphi\left(u_{0}+g(v),-v\right)$, which is closed but not contained in a vertical plane.

\section{References}

[Ale62] A. D. Alexandrov, A characteristic property of spheres. Ann. Mat. Pura Appl. (4) 58 (1962), 303-315. MR 143162

[AR04] U. Abresch and H. Rosenberg, A Hopf differential for constant mean curvature surfaces in $\mathbf{S}^{2} \times \mathbf{R}$ and $\mathbf{H}^{2} \times \mathbf{R}$. Acta Math. 193 (2) (2004), 141-174.Zbl 1078.53053 MR 2134864 
Vol. 82 (2007) Isometric immersions into 3-dimensional homogeneous manifolds

[AR05] U. Abresch and H. Rosenberg, Generalized Hopf differentials. Mat. Contemp. 28 (1) (2005), 1-28. Zbl 05031437 MR 2195187

[Bry87] R. Bryant, Surfaces of mean curvature one in hyperbolic space. Astérisque 154-155 (1988), 321-347. Zbl 0635.53047 MR 0955072

[Car92] M. do Carmo, Riemannian geometry. Math. Theory Appl., Birkhäuser, Boston, MA, 1992. Zbl 0752.53001 MR 1138207

[Dan04] B. Daniel, Isometric immersions into $\mathbb{S}^{n} \times \mathbb{R}$ and $\mathbb{H}^{n} \times \mathbb{R}$ and applications to minimal surfaces. Preprint, math.DG/0406426, 2004.

[Dan06] B. Daniel, Minimal disks bounded by three straight lines in Euclidean space and trinoids in hyperbolic space. J. Differential Geom. 72 (3) (2006), 467-508. Zbl 05039965 MR 2219941

[FMP99] C. Figueroa, F. Mercuri, and R. Pedrosa, Invariant surfaces of the Heisenberg groups. Ann. Mat. Pura Appl. (4) 177 (1999), 173-194. Zbl 0965.53042 MR 1747630

[GBKS03] K. Grosse-Brauckmann, R. Kusner, and J. Sullivan, Triunduloids: embedded constant mean curvature surfaces with three ends and genus zero. J. Reine Angew. Math. 564 (2003), 35-61. Zbl 1058.53005 MR 2021033

[Kar05] H. Karcher, Hyperbolic surfaces of constant mean curvature one with compact fundamental domains. In Global theory of minimal surfaces, Clay Math. Proc. 2, Amer. Math. Soc., Providence, RI, 2005, 311-323. MR 2167265

[Law70] H. B. Lawson, Complete minimal surfaces in $S^{3}$. Ann. of Math. (2) 92 (1970), 335-374. Zbl 0205.52001 MR 0270280

[NR04] B. Nelli and H. Rosenberg. Global properties of constant mean curvature surfaces in $\mathbb{H}^{2} \times \mathbb{R}$. Pacific J. Math., to appear.

[Pet98] P. Petersen, Riemannian geometry. Grad. Texts in Math. 171 Springer-Verlag, New York 1998. Zbl 0914.53001 MR 1480173

[Sco83] P. Scott, The geometries of 3-manifolds. Bull. London Math. Soc. 15 (5) (1983), 401-487. Zbl 0561.57001 MR 0705527

[SE05] R. Sá Earp, Parabolic and hyperbolic screw motion surfaces in $\mathbb{H}^{2} \times \mathbb{R}$. Preprint, PUC Rio, 2005.

[Ten71] K. Tenenblat, On isometric immersions of Riemannian manifolds. Bol. Soc. Brasil. Mat. 2 (2) (1971), 23-36. Zbl 0338.53010 MR 0328832

[UY93] M. Umehara and K. Yamada, Complete surfaces of constant mean curvature 1 in the hyperbolic 3-space. Ann. of Math. (2) 137 (3) (1993), 611-638. Zbl 0795.53006 MR 1217349

Received April 21, 2005

Benoît Daniel, IMPA, Estrada Dona Castorina 110, 22460-320 Rio de Janeiro - RJ, Brazil

E-mail: bdaniel@impa.br 